BNL-HET-01/18, CALT-68-2315

CERN-TH/2000-348

DCPT/01/56, IPPP/01/28

hep-ph/0106255

\title{
Implications of the Higgs boson searches on different soft SUSY-breaking scenarios
}

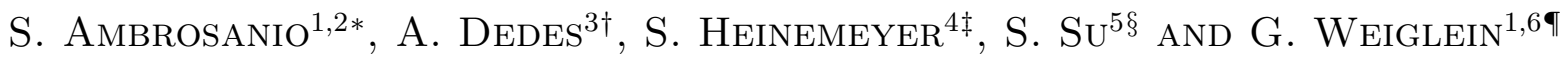 \\ ${ }^{1}$ CERN, TH Division, CH-1211 Geneva 23, Switzerland \\ ${ }^{2}$ Banca di Roma, Direzione Generale, Linea Finanza, \\ viale U. Tupini 180, I-00144 Roma, Italy (since Jan. 1, 2001) \\ ${ }^{3}$ Physikalisches Institut der Universität Bonn, Nußallee 12, D-53115 Bonn, Germany \\ ${ }^{4}$ HET, Physics Department, Brookhaven Natl. Lab., Upton, NY 11973, USA \\ ${ }^{5}$ California Institute of Technology, Pasadena, CA 91125, USA \\ ${ }^{6}$ Institute for Particle Physics Phenomenology, University of Durham, \\ Durham DH1 3LR, UK
}

\begin{abstract}
We investigate the Higgs boson sector of the Minimal Supersymmetric Standard Model (MSSM) in the framework of the three most prominent soft SUSY-breaking scenarios, mSUGRA, mGMSB and mAMSB. For each scenario, we determine the parameters at the electroweak scale from the set of input variables at higher energy scales (depending on the specific scenario) and evaluate the Higgs boson properties. The latter are based on results obtained within the Feynman-diagrammatic approach by taking into account the complete one-loop and the dominant two-loop contributions. The maximum value of the mass of the lightest neutral $\mathcal{C P}$-even MSSM Higgs boson, $m_{h}$, is determined in the three scenarios, and the behavior of the Higgs couplings to fermions and gauge bosons is investigated. Restrictions on $\tan \beta$ and on the set of higher-energy scale parameters are derived from the lower limits arising from the Higgs search at LEP2. We furthermore discuss the regions of parameter space in the three scenarios compatible with interpreting the excess observed at LEP2 as a Higgs signal, $m_{h}=115_{-0.9}^{+1.3} \mathrm{GeV}$. The case where the events observed at LEP2 could originate from the production of the heavier neutral $\mathcal{C P}$ even Higgs boson is also considered. The implications of a possible Higgs signal at $115 \mathrm{GeV}$ for SUSY searches at future colliders are briefly discussed for each of the three scenarios.
\end{abstract}

*email: Sandro.Ambrosanio@bancaroma.it

†email: dedes@th.physik.uni-bonn.de

$\ddagger$ email: Sven.Heinemeyer@bnl.gov

§email: shufang@theory.caltech.edu

๑email: Georg.Weiglein@cern.ch 


\section{Introduction}

The search for the light neutral Higgs boson is a crucial test of Supersymmetry (SUSY) that can be performed with the present and the next generation of high-energy colliders. The prediction of a relatively light Higgs boson is common to all supersymmetric models whose couplings remain in the perturbative regime up to a very high energy scale [1]. Finding the Higgs boson is thus one of the main goals of today's high-energy physics. The data taken during the final year of LEP running at $\sqrt{s} \gtrsim 206 \mathrm{GeV}$, while establishing a 95\% C.L. exclusion limit for the Standard Model (SM) Higgs boson of $m_{H}>113.5 \mathrm{GeV}$, showed at about the $3 \sigma$ level an excess of signal-like events over the background expectation which is in agreement with the expectation for the production of a SM Higgs boson of $m_{H}=115_{-0.9}^{+1.3} \mathrm{GeV}$ [2]. A Higgs mass value of about $115 \mathrm{GeV}$ would indicate that the SM can only be valid up to a scale $\Lambda \lesssim 10^{6} \mathrm{GeV}$ (or the vacuum must me meta stable), since new physics contributions are necessary in order to prevent the effective Higgs potential from becoming unstable [3]. In the Minimal Supersymmetric Standard Model (MSSM), on the other hand, the mass of the lightest $\mathcal{C} \mathcal{P}$-even Higgs boson, $m_{h}$, is bounded from above by $m_{h} \lesssim 135 \mathrm{GeV}$ 柾 (taking into account radiative corrections up

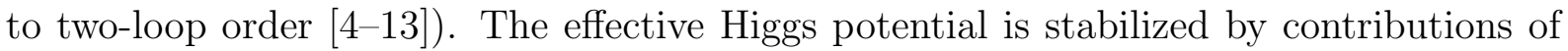
the Supersymmetric partners of the SM particles [14]. Within the MSSM, the LEP excess can be interpreted as the production of the lightest $\mathcal{C P}$-even Higgs boson, which over a wide parameter range has SM-like couplings, or of the heavier $\mathcal{C} \mathcal{P}$-even Higgs boson, in a region of parameter space where the $\mathcal{C P}$-odd Higgs boson $A$ is light and the ratio of the vacuum expectation values of the two Higgs doublets, $\tan \beta$, is relatively large.

In the MSSM no specific assumptions are made about the underlying Supersymmetry(SUSY)-breaking mechanism, and a parameterization of all possible SUSY-breaking terms is used. This gives rise to the huge number of more than 100 new parameters in addition to the SM, which in principle can be chosen independently of each other. A phenomenological analysis of this model in full generality would clearly be very involved, and one usually restricts to certain benchmark scenarios [15]. On the other hand, models in which all the low-energy parameters are determined in terms of a few parameters at the Grand Unification scale (or another high-energy scale), employing a specific soft SUSY-breaking scenario, are much more predictive. The most prominent scenarios in the literature are minimal Supergravity (mSUGRA) [16, 17, minimal Gauge Mediated SUSY Breaking (mGMSB) [18] and minimal Anomaly Mediated SUSY Breaking (mAMSB) [19 21]. Analyses of the Higgs sector in these scenarios have been performed [22 28], mostly focusing only on the maximum value of $m_{h}$. Within the mSUGRA scenario more recently some implications of the LEP2 results on the Higgs search have been investigated in the context of further constraints arising from the requirement that the lightest Supersymmetric particle (LSP) should give rise to an acceptable dark matter relic density, and that the predictions of the model should be in agreement with the experimental results on $b \rightarrow s \gamma$ and the anomalous magnetic moment of the muon.

In this paper we investigate in detail the predictions in the Higgs sector arising from the three SUSY-breaking scenarios mSUGRA, mGMSB and mAMSB. We relate the input from these scenarios in a uniform way to the predictions for the low-energy phenomenology

in the Higgs sector, allowing thus a direct comparison of the predictions arising from the 
different scenarios. The high-energy parameters given in the three scenarios are related to the low-energy SUSY parameters via renormalization group (RG) running, taking into account contributions up to two-loop order. After transforming the parameters obtained in this way into the corresponding on-shell parameters [29 31], they are used as input for the program FeynHiggs [32], which contains the complete one-loop and dominant twoloop corrections in the MSSM Higgs sector evaluated in the Feynman-diagrammatic (FD)

approach [4, 5, 33]. Further restrictions such as from precision observables and the nonobservation of SUSY particles are also taken into account. Based on these predictions for the Higgs sector phenomenology, we analyze the consequences of the results obtained from the Higgs search at LEP on the parameter space of the three scenarios. This is done by considering both the LEP exclusion bound [34] and the interpretation of the LEP excess as a possible signal. For the latter case we furthermore discuss the corresponding spectra of the SUSY particles in view of the SUSY searches at the next generation of colliders.

The rest of the paper is organized as follows. In Sect. 2 the three soft SUSY-breaking scenarios as well as the evaluation of the $\mathcal{C P}$-even Higgs boson sector of the MSSM are briefly reviewed. Details about the combination of renormalization-group equation (RGE) and FD calculation are given, and the parameter restrictions used are listed. The description of our data sets and the numerical analyses for the three scenarios is given in Sect. 3. The conclusions can be found in Sect. 4 .

\section{The Higgs sector in soft SUSY-breaking scenarios}

The fact that no SUSY partners of the SM particles have so far been observed means that low-energy SUSY cannot be realized as an unbroken symmetry in nature, and SUSY models thus have to incorporate extra supersymmetry breaking interactions. This is achieved by adding to the Lagrangian (defined by the given $\mathrm{SU}(3)_{C} \times \mathrm{SU}(2)_{L} \times \mathrm{U}(1)_{Y}$ gauge symmetry and the superpotential $W$ ) some extra interaction terms that respect the gauge symmetry but break supersymmetry. This breaking however should be such that no quadratic divergences appear and the technical "solution" to the hierarchy problem is not spoiled. Such terms are generally called "soft SUSY-breaking" terms. The most general supersymmetry breaking interaction Lagrangian resulting from spontaneously broken Supergravity in the flat limit $\left(M_{P} \rightarrow \infty\right.$, where $M_{P}$ is the Planck mass) contains just four types of soft SUSY-breaking terms [35], i.e. gaugino masses, $\Phi^{*} \Phi$-scalar masses, $\Phi \Phi \Phi$-scalar cubic superpotential interactions and $\Phi \Phi$-scalar quadratic superpotential interactions. Assuming that $R$-parity [36, 37] is conserved, which we do in this paper for all SUSY breaking scenarios, reduces the amount of new soft terms allowed in the Lagrangian. Choosing a particular soft SUSY-breaking pattern allows further reduction of the number of free parameters and the construction of predictive models.

In this section, we first explain how we employ the principle of radiative electroweak symmetry breaking (REWSB). Then we introduce the three most commonly studied soft SUSY-breaking scenarios and describe the general method used to derive predictions for the low-energy Higgs sector, which applies to all scenarios. 


\subsection{Radiative electroweak symmetry breaking}

The investigation of REWSB in the MSSM [38] relies on a RG analysis. The Higgs boson "running" mass-squared matrix, although positive definite at large energy scales of the order of the Grand Unification scale $M_{\mathrm{GUT}}$, yields a negative eigenvalue at low energies causing the spontaneous breakdown of the electroweak (EW) symmetry. The result can be interpreted as a prediction of $M_{Z}$ in terms of parameters at a large energy scale. Alternatively, one can consider $M_{Z}$ as being determined by experiment and derive in this way the absolute value of the $\mu$-parameter (which defines the coupling of the two Higgs doublets) as well as the value of the bilinear soft-SUSY breaking parameter $B$ at a scale in the vicinity of the EW scale, from the minimization conditions of the effective potential,

$$
\begin{aligned}
\mu^{2}(Q) & =\frac{\bar{m}_{H_{1}}^{2}-\bar{m}_{H_{2}}^{2} \tan ^{2} \beta}{\tan ^{2} \beta-1}-\frac{1}{2} M_{Z}^{2}(Q), \\
B(Q) & =-\frac{\left(\bar{m}_{1}^{2}+\bar{m}_{2}^{2}\right) \sin 2 \beta}{2 \mu(Q)},
\end{aligned}
$$

where $Q$ is derived from the scalar fermion sector. It is usually chosen such that radiative corrections to the effective potential are rather small compared to other scales. In eqs. (1), (2) $\tan \beta \equiv v_{2} / v_{1}$ is the ratio of the two vacuum expectation values of the Higgs fields $H_{2}$ and $H_{1}$ responsible for giving masses to the up-type and down-type quarks, respectively. In eqs. (11),(2), $\tan \beta$ is evaluated at the scale $Q$, from the scale $M_{Z}$, where it is considered as an input parameter". By $\bar{m}_{H_{i}}^{2}=m_{H_{i}}^{2}+\Sigma_{v_{i}}$ in eqs. (11),(21) we denote the radiatively corrected "running" Higgs soft-SUSY breaking masses and

$$
\bar{m}_{i}^{2}=m_{H_{i}}^{2}+\mu^{2}+\Sigma_{v_{i}} \equiv \bar{m}_{H_{i}}^{2}+\mu^{2} \quad(i=1,2),
$$

where $\Sigma_{v_{i}}$ are the one-loop corrections based on the 1-loop Coleman-Weinberg effective potential $\Delta V, \Sigma_{v_{i}}=\frac{1}{2 v_{i}} \frac{\partial \Delta V}{\partial v_{i}}$,

$$
\Sigma_{v_{i}}=\frac{1}{64 \pi^{2}} \sum_{a}(-)^{2 J_{a}}\left(2 J_{a}+1\right) C_{a} \Omega_{a} \frac{M_{a}^{2}}{v_{i}} \frac{\partial M_{a}^{2}}{\partial v_{i}}\left[\ln \frac{M_{a}^{2}}{Q^{2}}-1\right] .
$$

Here $J_{a}$ is the spin of the particle $a, C_{a}$ are the color degrees of freedom, and $\Omega_{a}=1(2)$ for real scalar (complex scalar), $\Omega_{a}=1(2)$ for Majorana (Dirac) fermions. $Q$ is the energy scale and the $M_{a}$ are the field dependent mass matrices. Explicit formulas of the $\Sigma_{v_{i}}$ are given in the Appendices of Refs. [40,41]. In our analyses contributions from all SUSY particles at the one-loop level are incorporated?. With $M_{Z}^{2}$ here we denote the tree level "running" $Z$ boson mass, $M_{Z}^{2}(Q)=\frac{1}{2}\left(g_{1}^{2}+g_{2}^{2}\right) v^{2}\left(v^{2} \equiv v_{1}^{2}+v_{2}^{2}\right)$, extracted at the scale $Q$ from its physical pole mass $M_{Z}=91.187 \mathrm{GeV}$. The REWSB is fulfilled, see Sect. 2.6.3, if and only if there is a solution to the eqs. (四),(22) ? $^{\text {. }}$.

\footnotetext{
${ }^{1}$ See for example the discussion in the Appendix of Ref. [39] or in Ref. [6].

2 The corresponding two-loop corrections are not available yet. Assuming the size of these unknown higher-order corrections to be of the same size as for the Higgs-boson mass matrix, see Sect. 2.5, the resulting values of $\mu$ and $B$ could change by $\sim 5-10 \%$. These parameters will serve as input for our numerical analysis in Sect. 3. The possible changes would hardly affect our results obtained in the Higgs-boson sector and only mildly affect the analysis of SUSY particle spectra in Sect. 3.5.

3 Sometimes in the literature, the requirement of the REWSB is described by the inequality $m_{1}^{2}(Q) m_{2}^{2}(Q)-|\mu(Q) B(Q)|^{2}<0$. This relation is automatically satisfied here from eqs. (1),(2) and from the fact that the physical squared Higgs masses must be positive.
} 


\section{2 mSUGRA}

A dramatic simplification of the structure of the SUSY breaking interactions is provided either by Grand Unification assumptions or by Superstrings. For example, SU(5) unification implies at tree level equality relations between the scalar soft-SUSY breaking masses $m_{\tilde{Q}}=m_{\tilde{U}^{c}}=m_{\tilde{E}^{c}}$, and $m_{\tilde{L}}=m_{\tilde{D}^{c}}$, equality between the soft breaking gaugino masses $M_{1}=M_{2}=M_{3}$ and for two of the trilinear soft breaking couplings, $A_{d}=A_{e}$. On the other hand, $\mathrm{SO}(10)$ unification implies equality of all scalar particle masses, equality of Higgs masses and equality of the three types of trilinear couplings. The simplest possible choice at tree level is to take all scalar particle and Higgs masses equal to a common mass parameter $M_{0}$, all gaugino masses are chosen to be equal to the parameter $M_{1 / 2}$ and all trilinear couplings flavor blind and equal to $A_{0}$. This situation is common in the effective Supergravity theories resulting from Superstrings but there exist more complicated alternatives. Interestingly, the contribution of the family-anomalous U(1) universal D-term to the scalar quark masses may be intra-family non-universal, and may differ from the usually assumed universal boundary conditions [42]. Another alternative are for example Superstrings with massless string modes of different modular weights that lead to different scalar particle masses at tree level [43]. Thus, it seems that in almost all the "realistic" models motivated by Grand Unified Theories (GUTs) or Superstrings the universality assumption is broken and each of these models has to be addressed separately in order to study its phenomenology at low energies. On the other hand, one should note that such non-minimal alternatives like flavor-dependent scalar particle masses are constrained by limits on Flavor Changing Neutral Currents (FCNC) processes. In what follows, we shall therefore consider the simplest (and most commonly used) case of the three parameters at the GUT scale, namely $M_{0}, M_{1 / 2}$ and $A_{0}$, which is usually called the mSUGRA scenario.

In order to solve eqs. (11),(2), i.e. in order to impose the constraint of REWSB, one needs as input $\tan \beta\left(M_{Z}\right)$ and $\operatorname{sign}(\mu)$. The running soft SUSY-breaking Higgs mass parameters, $m_{H_{1}}$ and $m_{H_{2}}$, are defined at the EW scale after their evolution from the GUT scale where we assume that they have a common value, $M_{0}$. In addition the radiative corrections $\Sigma_{v_{i}}$ to the minimization conditions eq. (田) are defined from the low-energy SUSY spectrum and the masses of the SM particles, which in turn means knowledge of $M_{0}, M_{1 / 2}$ and $A_{0}$ at the GUT scale. Thus, apart from the SM masses provided by the experimental data [44], 4 parameters and a sign are required to define the mSUGRA scenario:

$$
\left\{M_{0}, M_{1 / 2}, A_{0}, \tan \beta, \operatorname{sign}(\mu)\right\} .
$$

In our numerical procedure we employ a two-loop renormalization group analysis for all parameters involved, i.e. all couplings, dimensionful parameters and VEV's. We start with the $\overline{\mathrm{MS}}$ values for the gauge couplings at the scale $M_{Z}$, where for the strong coupling constant $\alpha_{s}$ a trial input value in the vicinity of 0.120 is used. The $\overline{\mathrm{MS}}$ values are converted into the corresponding $\overline{\mathrm{DR}}$ ones [45]. The $\overline{\mathrm{MS}}$ running $b$ and $\tau$ masses are run down to $m_{b}=4.9 \mathrm{GeV}, m_{\tau}=1.777 \mathrm{GeV}$ with the $\mathrm{SU}(3)_{C} \times \mathrm{U}(1)_{\text {em }}$ RGE's 46] to derive the running bottom and tau masses (extracted from their pole masses). This procedure includes all SUSY corrections at the one-loop level and all QCD corrections at the twoloop level as given in Ref. [41]. Afterwards by making use of the two-loop RGE's for 
the running masses $\bar{m}_{b}, \bar{m}_{\tau}$, we run upwards to derive their $\overline{\mathrm{MS}}$ values at $M_{Z}$, which are subsequently converted to the corresponding $\overline{\mathrm{DR}}$ values. This procedure provides the bottom and tau Yukawa couplings at the scale $M_{Z}$. The top Yukawa coupling is derived from the top-quark pole mass, $m_{t}=175 \mathrm{GeV}$, which is subsequently converted to the $\overline{\mathrm{DR}}$ value, $\overline{m_{t}}\left(m_{t}\right)$, where the top Yukawa coupling is defined. The evolution of all couplings from $M_{Z}$ running upwards to high energies now determines the unification scale $M_{\mathrm{GUT}}$ and the value of the unification coupling $\alpha_{\mathrm{GUT}}$ by

$$
\left.\alpha_{1}\left(M_{\mathrm{GUT}}\right)\right|_{\overline{\mathrm{DR}}}=\left.\alpha_{2}\left(M_{\mathrm{GUT}}\right)\right|_{\overline{\mathrm{DR}}}=\alpha_{\mathrm{GUT}} .
$$

At the GUT scale we set the boundary conditions for the soft SUSY breaking parameters, i.e. the values for $M_{0}, M_{1 / 2}$ and $A_{0}$ are chosen, and also $\alpha_{3}\left(M_{\mathrm{GUT}}\right)$ is set equal to $\alpha_{\mathrm{GUT}}$. All parameters are run down again from $M_{\mathrm{GUT}}$ to $M_{Z}$. For the calculation of the soft SUSY-breaking masses at the EW scale we use the "step function approximation" 47. Thus, if the equation employed is the RGE for a particular running mass $m(Q)$, then $Q_{0}$ is the corresponding physical mass determined by the condition $m\left(Q_{0}\right)=Q_{0}$. After running down to $M_{Z}$, the trial input value for $\alpha_{s}$ has changed. At this point the value for $\tan \beta$ is chosen and fixed. As described in Sect. 2.1, the parameters $|\mu|$ and $B$ are calculated from the minimization conditions (11) and (2), respectively. Only the sign of the $\mu$-parameter is not automatically fixed and thus chosen now. This procedure is iterated several times until convergence is reached.

\section{3 mGMSB}

A very promising alternative to mSUGRA is based on the hypothesis that the soft SUSY breaking (SSB) occurs at relatively low energy scales and it is mediated mainly by gauge interactions through the so-called "messenger sector" (GMSB) 18, 48, 49. This scheme provides a natural, automatic suppression of the SUSY contributions to flavor-changing neutral currents and $\mathcal{C} \mathcal{P}$-violating processes. Furthermore, in the simplest versions of GMSB (denoted hereafter with mGMSB), the MSSM spectrum and most of the observables depend on just 4 parameters and a sign,

$$
\left\{M_{\text {mess }}, N_{\text {mess }}, \Lambda, \tan \beta, \operatorname{sign}(\mu)\right\},
$$

where $M_{\text {mess }}$ is the overall messenger mass scale; $N_{\text {mess }}$ is a number called the messenger index, parameterizing the structure of the messenger sector; $\Lambda$ is the universal soft SUSY-breaking mass scale felt by the low-energy sector; $\tan \beta$ is the ratio of the vacuum expectation values of the two Higgs doublets; $\operatorname{sign}(\mu)$ is the ambiguity left for the SUSY higgsino mass after imposing a correct REWSB (see Sect. 2.1 and e.g. Refs. [22, 50, 53]).

The phenomenology of GMSB (and more in general of any theory with low-energy $\mathrm{SSB}$ ) is characterized by the presence of a very light gravitino $\tilde{G}$ with mass [54 given by $m_{3 / 2}=m_{\tilde{G}}=\frac{F}{\sqrt{3} M_{P}^{\prime}} \simeq\left(\frac{\sqrt{F}}{100 \mathrm{TeV}}\right)^{2} 2.37 \mathrm{eV}$, where $\sqrt{F}$ is the fundamental scale of SSB and $M_{P}^{\prime}=2.44 \times 10^{18} \mathrm{GeV}$ is the reduced Planck mass. Since $\sqrt{F}$ is typically of order $100 \mathrm{TeV}$, the $\tilde{G}$ is always the LSP in these theories. Hence, if $R$-parity is conserved, any MSSM particle will decay into the gravitino. Depending on $\sqrt{F}$, the interactions of the gravitino, although much weaker than gauge and Yukawa interactions, can still be strong 
enough to be of relevance for collider physics. In most cases, the last step of any SUSY decay chain is the decay of the next-to-lightest SUSY particle (NLSP), which can occur either outside or inside a typical detector, possibly close to the interaction point. The nature of the NLSP - or, more precisely, of the SUSY particle(s) having a large branching ratio for decaying into the gravitino and the relevant SM partner - determines four main scenarios giving rise to qualitatively different phenomenology [51].

The low-energy parameter sets for this scenario have been calculated by using the program SUSYFIRE [55] and adopting the phenomenological approach of Refs. [51 53], see also Ref. [27]. The origin of $\mu$ is not specified, nor the assumption $B \mu=0$ is made at the messenger scale. Instead, correct REWSB is imposed to trade $\mu$ and $B \mu$ for $M_{Z}$ and $\tan \beta$, leaving the sign of $\mu$ undetermined, see Sect. 2.1. However, note that to build a fully coherent GMSB model, one should also find a more fundamental solution to the latter problem, perhaps providing a dynamical mechanism to generate $\mu$ and $B \mu$, possibly with values of the same order of magnitude. This might be accomplished radiatively through some new interaction. In this case, the other soft terms in the Higgs potential, namely $m_{H_{1,2}}^{2}$, will be also affected and this will in turn change the values of $|\mu|$ and $B \mu$ coming from REWSB conditions. We have checked this circumstance in detail in the case where one has an extra term in the Higgs potential of the type $\Delta_{+}$(see the parameterization of Ref. [50]) and also performed some checks in the general case. In all cases we did not find any big changes in $m_{h}$.

To determine the MSSM spectrum and low-energy parameters, the RGE evolution is solved with boundary conditions at the $M_{\text {mess }}$ scale, where

$$
\begin{aligned}
& M_{a}\left(M_{\text {mess }}\right)=N_{\text {mess }} \Lambda g\left(\frac{\Lambda}{M_{\text {mess }}}\right) \alpha_{a}, \quad(a=1,2,3) \\
& \tilde{m}^{2}\left(M_{\text {mess }}\right)=2 N_{\text {mess }} \Lambda^{2} f\left(\frac{\Lambda}{M_{\text {mess }}}\right) \sum_{a}\left(\frac{\alpha_{a}}{4 \pi}\right)^{2} C_{a},
\end{aligned}
$$

for the gaugino and the scalar masses, respectively. The exact expressions for $g$ and $f$ at the one- and two-loop level can be found, e.g., in Ref. [51], and $C_{a}$ are the quadratic Casimir invariants for the scalar fields. As usual, the scalar trilinear couplings $A_{f}$ are assumed to vanish at the messenger scale, as suggested by the fact that they (and not their square) are generated via gauge interactions with the messenger fields at the two loop-level only.

The interesting region of the GMSB parameter space is selected as follows. Barring the case where a neutralino is the NLSP and decays outside the detector (large $\sqrt{F}$ ), the GMSB signatures are very spectacular and the SM background is generally negligible or easily subtractable. Therefore, also in accordance with negative results in the LEP2 searches [56], only models where the NLSP mass is larger than $100 \mathrm{GeV}$ are considered. Other requirements are: $M_{\text {mess }}>1.01 \Lambda$, to prevent an excess of fine-tuning of the messenger masses; the mass of the lightest messenger scalar be at least $10 \mathrm{TeV}$; $M_{\text {mess }}>M_{\mathrm{GUT}} \times \exp \left(-125 / N_{\text {mess }}\right)$, to ensure the perturbativity of gauge interactions up to the GUT scale; $M_{\text {mess }} \lesssim 10^{5} \Lambda$, for simplicity. As a result, the messenger index $N_{\text {mess }}$, which is assumed to be an integer independent of the gauge group, cannot be larger than 8 . To prevent the top Yukawa coupling from blowing up below the GUT scale, $\tan \beta>1.5$ 
is required. Models with $\tan \beta \gtrsim 55$ (with a mild dependence on $\Lambda$ ) are forbidden by the REWSB requirement, see Sect. 2.6.3, and typically fail to give $M_{A}^{2}>0$.

The models are generated using SUSYFIRE with the following prescriptions for the high-energy input parameters. Logarithmic steps have been used for $\Lambda$ (between about $45 \mathrm{TeV} / N_{\text {mess }}$ and about $220 \mathrm{TeV} / \sqrt{N_{\text {mess }}}$ ), $M_{\text {mess }} / \Lambda$ (between about 1.01 and $10^{5}$ ) and $\tan \beta$ (between 1.5 and about 60 ), subject to the constraints described above. SUSYFIRE starts from the values of particle masses and gauge couplings at the weak scale and then evolves them up to the messenger scale through RGEs. At the messenger scale, it imposes the boundary conditions (8) for the soft particle masses and then evolves the RGEs back to the electroweak scale. The decoupling of each SUSY particle at the proper threshold is taken into account. Two-loop RGEs are used for gauge couplings, third generation Yukawa couplings and gaugino soft masses. The other RGEs are taken at the one-loop levelf. At the scale $Q$, derived from the scalar fermion sector, REWSB conditions are imposed by means of the one-loop effective potential approach. For the $\Sigma_{v_{i}}$ in eq. (田) all dominant corrections from the stop, sbottom and stau sector are included. The program then evolves up again to $M_{\text {mess }}$ and so on. Three or four iterations are usually enough to get a good approximation for the MSSM spectrum.

\section{4 mAMSB}

The most recently proposed Anomaly Mediated SUSY Breaking (AMSB) scenario [19,20 provides an alternative way to give mass to all the SUSY particles. In this model, SUSY breaking happens on a separate brane and is communicated to the visible world via the super-Weyl anomaly. The overall scale of SUSY particle masses is set by $m_{\text {aux }}$, which is the VEV of the auxiliary field in the supergravity multiplet. In the AMSB scenario, the low-energy soft supersymmetry breaking parameters $M_{i}$ (gaugino masses, $i=1-3$ ), $m_{\text {scalar }}^{2}$ and $A_{y}$ at the GUT scale are given by [19,21]

$$
\begin{aligned}
M_{i} & =\frac{\beta_{g_{i}}}{g_{i}} m_{\mathrm{aux}} \\
m_{\mathrm{scalar}}^{2} & =-\frac{1}{4}\left(\frac{\partial \gamma}{\partial g} \beta_{g}+\frac{\partial \gamma}{\partial y} \beta_{y}\right) m_{\mathrm{aux}}^{2}+m_{0}^{2} \\
A_{y} & =-\frac{\beta_{y}}{y} m_{\mathrm{aux}} .
\end{aligned}
$$

Notice that the slepton squared-masses would be negative if $m_{0}$ were absent. There have been several proposals to solve this tachyonic slepton problem: bulk contributions [19, non-decoupling effects of ultra-heavy vectorlike matter fields [57, coupling of extra Higgs doublets to the leptons [58], contributions from the $R$-parity violating couplings in eq. (10) (with $m_{0}=0$ ) [59, and a heavy mass threshold contribution at higher orders 60. Here we have adopted a phenomenological approach and have introduced an additional

${ }^{4}$ Contrary to the mSUGRA scenario in Sect. 2.2 the scalar masses are treated only at the one-loop level in mGMSB and the mAMSB scenario in Sect. 2.4. However, the main effects arise from the Yukawa couplings, which are consistently treated at the two-loop level. The two-loop effects on the scalar masses have been shown to be at the $5 \%$ level [47. 
mass scale $m_{0}$ at the GUT scale in order to keep the slepton masses positive [21]. For simplification, we choose $m_{0}$ to be the same for all the super scalar particles. Therefore, in the minimal case (mAMSB), the particle spectrum can be determined by 3 parameters and a sign:

$$
\left\{m_{\mathrm{aux}}, m_{0}, \tan \beta, \operatorname{sign}(\mu)\right\} .
$$

Eqs. (9), (10) and (11) would hold at all scales if $m_{0}$ were absent. However, once $m_{0}$ is introduced at the GUT scale, the above definitions of $M_{i}, m_{\text {scalar }}^{2}$ and $A_{y}$ set the boundary conditions and the entire SUSY spectrum can be obtained via the running of supersymmetric RGEs down to a lower scale.

Once the squark threshold is crossed, the squarks decouple and one is left with an effective field theory with two Higgs doublets and all the standard model particles?. The two unknown parameters $|\mu|$ and $B$ are determined by the minimization of the Higgs effective potential as explained in Sect. 2.1. Therefore, the low-energy spectrum is fixed once the values of $m_{\mathrm{aux}}, m_{0}, \tan \beta$ and the sign of $\mu$ are known.

\subsection{Evaluation of predictions in the Higgs boson sector of the MSSM}

The most relevant parameters for Higgs boson phenomenology in the MSSM are the mass of the $\mathcal{C P}$-odd Higgs boson, $M_{A}$, the ratio of the two vacuum expectation values, $\tan \beta$, the scalar top masses and mixing angle, $m_{\tilde{t}_{1}}, m_{\tilde{t}_{2}}, \theta_{\tilde{t}}$, for large $\tan \beta$ also the scalar bottom masses and mixing angle, $m_{\tilde{b}_{1}}, m_{\tilde{b}_{2}}, \theta_{\tilde{b}}$, the Higgs mixing parameter, $\mu$, the gluino mass, $m_{\tilde{g}}$, and the $\mathrm{U}(1)$ and $\mathrm{SU}(2)$ gaugino masses, $M_{1}$ and $M_{2}$. The way in which these low-energy parameters are derived in each of the soft SUSY-breaking scenarios has been described in Sects. 2.2 - 2.4. Since the RG running employed in the three scenarios is based on the $\overline{\mathrm{DR}}$ scheme, the corresponding low-energy parameters are $\overline{\mathrm{DR}}$ parameters. In order to derive predictions for observables, i.e. particle masses and mixing angles, these parameters in general have to be converted into on-shell parameters.

For the predictions in the MSSM Higgs sector we use results obtained in the Feynmandiagrammatic (FD) approach (see below) within the on-shell renormalization scheme. Since they incorporate two-loop contributions in the $t-\tilde{t}$ sector, the parameters in the scalar top sector (which enter at one-loop order in the predictions for the Higgs boson masses) have to be appropriately converted from $\overline{\mathrm{DR}}$ to on-shell parameters 29. 31. We perform this conversion using the full $\mathcal{O}\left(\alpha_{s}\right)$ contributions.

In the FD approach the masses of the two $\mathcal{C P}$-even Higgs bosons, $m_{h}$ and $m_{H}$, are derived beyond tree level by determining the poles of the $h-H$-propagator matrix whose inverse is given by

$$
\left(\Delta_{\text {Higgs }}\right)^{-1}=-i\left(\begin{array}{cc}
q^{2}-m_{H, \text { tree }}^{2}+\hat{\Sigma}_{H}\left(q^{2}\right) & \hat{\Sigma}_{h H}\left(q^{2}\right) \\
\hat{\Sigma}_{h H}\left(q^{2}\right) & q^{2}-m_{h, \text { tree }}^{2}+\hat{\Sigma}_{h}\left(q^{2}\right)
\end{array}\right),
$$

\footnotetext{
${ }^{5}$ Gluinos are also decoupled since their masses are close to the squark masses. The contributions of Bino and Winos to the Higgs sector can be neglected since the $\mathrm{U}(1)_{Y}$ and $\mathrm{SU}(2)$ gauge couplings are small.
} 
where the $\hat{\Sigma}$ denote the renormalized Higgs boson self-energies. Determining the poles of the matrix $\Delta_{\text {Higgs }}$ in eq. (13) is equivalent to solving the equation

$$
\left[q^{2}-m_{h, \text { tree }}^{2}+\hat{\Sigma}_{h}\left(q^{2}\right)\right]\left[q^{2}-m_{H, \text { tree }}^{2}+\hat{\Sigma}_{H}\left(q^{2}\right)\right]-\left[\hat{\Sigma}_{h H}\left(q^{2}\right)\right]^{2}=0 .
$$

We use the result for the Higgs boson self-energies consisting of the complete one-loop result for the Higgs boson self-energies in the on-shell scheme [33] combined with the dominant two-loop contributions of $\mathcal{O}\left(\alpha \alpha_{s}\right)$ [4, 5] and further subdominant corrections $\square$, 8], see Ref. [4] for details. The matrix eq. (13) therefore contains the renormalized Higgs boson self-energies

$$
\hat{\Sigma}_{s}\left(q^{2}\right)=\hat{\Sigma}_{s}^{(1)}\left(q^{2}\right)+\hat{\Sigma}_{s}^{(2)}(0), \quad s=h, H, h H,
$$

where the momentum dependence is neglected only in the two-loop contribution.

An effective mixing angle,

$$
\alpha_{\mathrm{eff}}=\arctan \left[\frac{-\left(M_{A}^{2}+M_{Z}^{2}\right) \sin \beta \cos \beta-\hat{\Sigma}_{\phi_{1} \phi_{2}}}{M_{Z}^{2} \cos ^{2} \beta+M_{A}^{2} \sin ^{2} \beta-\hat{\Sigma}_{\phi_{1}}-m_{h}^{2}}\right], \quad-\frac{\pi}{2}<\alpha_{\mathrm{eff}}<\frac{\pi}{2},
$$

can furthermore be obtained from diagonalizing the mixing matrix in the basis of the unrotated neutral $\mathcal{C P}$-even fields $\phi_{1}, \phi_{2}$, neglecting the momentum dependence everywhere (alternatively, the mass matrix in the $h, H$ basis is diagonalized by the angle $\Delta \alpha$, where $\alpha_{\text {eff }}=\alpha_{\text {tree }}+\Delta \alpha$, see e.g. Ref. [61]). Inserting $\alpha_{\text {eff }}$ in the tree-level formulas for Higgs production and decay, the dominant universal corrections in the Higgs sector are taken into account 61, 62].

The results for the Higgs boson masses and $\alpha_{\text {eff }}$ as well as the conversion from $\overline{\mathrm{DR}}$ to on-shell parameters using the full $\mathcal{O}\left(\alpha_{s}\right)$ contributions for both the parameters in the $t-\tilde{t}$ and $b-\tilde{b}$ sector are implemented in the Fortran code FeynHiggs [32].

For the investigation of the mSUGRA scenario FeynHiggs has been interfaced to the program SUITY 63], used for the evaluation of the low-energy spectrum of the mSUGRA scenario. A combined program, FeynSSG [64], has been created on the basis of FeynHiggs and SUITY, in which the two subprograms run automatically.

As a further check of our results for the Higgs boson sector, we have (in addition to the FeynHiggs calculation) evaluated all results with a code based on an independent oneloop calculation [65], but using the two-loop routines of FeynHiggs. The difference of the one-loop calculation based on Ref. [33, used in FeynHiggs, and the ones given in Ref. 65] are only due to different renormalization prescriptions and thus of higher order [66]. The results we found in both approaches are as expected very similar and lead to the same conclusions.

\subsection{Other constraints}

While our main focus in this paper is on the physics in the Higgs sector, we also take into account some further (relatively mild) constraints when determining the allowed parameter values. These constraints are discussed in the following. 


\subsubsection{Precision observables}

The electroweak precision observables are affected by the whole spectrum of SUSY particles. The main SUSY contributions to the $W$ boson mass, $M_{W}$, the effective leptonic weak mixing angle, $\sin ^{2} \theta_{\text {eff }}$, and other $Z$ boson observables usually arise from $\tilde{t} / \tilde{b}$ contributions. They enter via the leading contribution to the $\rho$-parameter [67]. In our analysis we take into account the corrections arising from $\tilde{t} / \tilde{b}$ loops up to two-loop order [68]. A value of $\Delta \rho$ outside the experimentally preferred region of $\Delta \rho^{\text {SUSY }} \lesssim 3 \times 10^{-3}$ [44] indicates experimentally disfavored $\tilde{t}$ and $\tilde{b}$ masses $\sigma$. The evaluation of $\Delta \rho^{\mathrm{SUSY}}$ is implemented in FeynHiggs.

We have verified that in our analysis below the $\Delta \rho$ constraint does not play a significant role, i.e. nearly all generated model points give rise to an acceptable contribution to the electroweak precision observables. As a conservative approach, we do not apply any further constraints from $g_{\mu}-2$ or $b \rightarrow s \gamma$.

\subsubsection{Experimental bounds on SUSY particle masses}

The search for SUSY particles has been one of the main tasks pursued at Run I of the Tevatron and at LEP. The searches all turned out to be negative, thus lower limits on the SUSY particle masses have been set. In order to restrict the allowed parameter space in the three soft SUSY-breaking scenarios we employed the following constraints on their low-energy mass spectrum [44, 56, 69 72]:

$$
\begin{aligned}
& m_{\tilde{e}}>95 \mathrm{GeV} \text { (mSUGRA, AMSB) } \\
& m_{\tilde{\mu}}>85 \mathrm{GeV} \text { (mSUGRA, AMSB) } \\
& m_{\tilde{\tau}}>71 \mathrm{GeV} \text { (mSUGRA, AMSB) } \\
& m_{\tilde{\nu}}>43 \mathrm{GeV} \text { (mSUGRA, AMSB) } \\
& m_{\tilde{t}}>95 \mathrm{GeV} \text { (mSUGRA, AMSB) } \\
& m_{\tilde{b}}>85 \mathrm{GeV} \text { (mSUGRA, AMSB) } \\
& m_{\tilde{g}}>190 \mathrm{GeV} \text { (mSUGRA, AMSB) } \\
& m_{\tilde{\chi}^{ \pm}}>103 \mathrm{GeV}\left(\mathrm{mSUGRA}, m_{\tilde{\nu}}>300 \mathrm{GeV}\right) \\
& m_{\tilde{\chi}^{ \pm}} \gtrsim 84.6 \mathrm{GeV}\left(\mathrm{mSUGRA}, m_{\tilde{\nu}}<300 \mathrm{GeV}\right) \\
& m_{\tilde{\chi}^{ \pm}} \gtrsim 45 \mathrm{GeV} \text { (AMSB) } \\
& m_{\tilde{\chi}_{1}^{0}} \gtrsim 36 \mathrm{GeV} \text { (mSUGRA) } \\
& m_{\tilde{\chi}_{1}^{0}} \gtrsim 45 \mathrm{GeV} \text { (AMSB) } \\
& m_{\mathrm{NLSP}}>100 \mathrm{GeV} \text { (GMSB). }
\end{aligned}
$$

Note that the NLSP condition in the GMSB scenario (applying either to the lightest neutralino or to the lighter stau) automatically imposes stronger bounds on the other particle masses than the purely experimental bounds.

\footnotetext{
${ }^{6}$ Since the $\Delta \rho^{\text {SUSY }}$ evaluation involves scalar bottoms at the two-loop level, also the parameters in the $\tilde{b}$ sector have to be transformed from $\overline{\mathrm{DR}}$ to on-shell.
} 


\subsubsection{Other phenomenological restrictions}

Besides constraints from precision observables and from unsuccessful direct search for SUSY particles, we also take into account the following restrictions (if not indicated otherwise):

- For the top-quark mass, throughout this paper we use the value $m_{t}=175 \mathrm{GeV}$. A variation of $m_{t}$ directly affects the result for $m_{h}$, while its influence on the other quantities studied here is more moderate. As a rule of thumb, a change in $m_{t}$ by $\pm 1 \mathrm{GeV}$ also results in a change in $m_{h}$ of about $\pm 1 \mathrm{GeV}$ [73].

- The GUT or high-energy scale parameters are taken to be real, no SUSY $\mathcal{C P}$ violating phases are assumed.

- In all models under consideration the $R$-parity symmetry [36, 37] is taken to be conserved.

- Parameter sets that do not fulfill the condition of radiative electroweak symmetry breaking (REWSB), i.e. the one-loop minimization conditions of eqs. (1),(2), are discarded (already at the level of model generation.)

Within all soft SUSY-breaking scenarios considered here, the condition of REWSB leads to restrictions on $\tan \beta$. For example, it is almost impossible (or a huge fine tuning is required) to find very large values of $\tan \beta, \tan \beta \gtrsim 60$ which pass this constraint. This is because in that region both the RGEs of the Higgs soft breaking masses receive large corrections not only from the top Yukawa coupling but also from the bottom and the tau Yukawa couplings. This drives the numerator (and thus $\mu^{2}$ ) of eq. (11) negative, thus excluding this parameter set from our analysis. Another possibility of not fulfilling the REWSB condition is a very heavy soft SUSYbreaking spectrum, so that $m_{H_{2}}^{2}$ does not reach negative values and thus does not trigger REWSB.

- Parameter sets that do not fulfill the "strong CCB" constraints are discarded (already at the level of model generation), i.e. models for which the physical vacuum would be charge or color breaking. In our analysis this corresponds to cases where the squared scalar quark or charged lepton masses are becoming negative at the scale $Q$, where $Q$ is the energy scale at which the low-energy parameters are decoupled. However, we do not test the models for local or global charge or color breaking minima in general. In most cases the tunneling time from our charge and color conserving vacuum to the charge or color breaking minimum is much longer than the present age of the universe, and thus they are in practice not dangerous [74 77].

- The original motivation for the introduction of SUSY into particle physics was the solution of the "hierarchy problem". This sets a natural upper bound on the SUSY particle masses, which of course depends on how much fine tuning one is willing to accept. In our analysis we have imposed a (rather mild) "naturalness bound". This upper bound on the SUSY particle masses has been chosen to be equal for all 
three soft SUSY-breaking scenarios. Thus the low-energy mass spectra are directly comparable. We have imposed

$$
m_{\tilde{q}} \lesssim 1.5 \mathrm{TeV}, \quad m_{\tilde{g}} \lesssim 2 \mathrm{TeV} .
$$

These bounds give rise to upper bounds also on the other scalar masses and on the electroweak gaugino masses (depending on the specific scenario).

The bounds imposed in eq. (18) can of course not be considered as strict upper bounds (although constraints from cosmology, $b \rightarrow s \gamma$ and $g_{\mu}-2$ in general also favor a relatively light particle spectrum [78 81]), but carry a certain degree of arbitrariness. In particular, we do not consider here the scenario of focus point supersymmetry [82, in which squarks and sleptons in the multi-TeV range can occur. It should be noted, however, that in all three soft SUSY-breaking scenarios an upper bound of $\mathcal{O}(5 \mathrm{TeV})$ is obtained by the requirement of REWSB. On the other hand, saturating this upper bound of $\mathcal{O}(5 \mathrm{TeV})$ requires severe fine tuning to satisfy the minimization conditions given in eqs. (11), (2).

The imposed upper bounds on SUSY masses also naturally result in a limit for the soft SUSY-breaking parameters at the high energy scale, $M_{0}, M_{1 / 2}$ in mSUGRA, $\Lambda$ in mGMSB and $m_{0}, m_{\text {aux }}$ in mAMSB. If the bounds in eq. (18) were relaxed, heavier particle spectra would be allowed. The effect on the Higgs boson sector is only logarithmic and thus rather small. Concerning the collider phenomenology as presented in Sect. 3.5, the detection of SUSY particles would become more difficult.

- We demand that the lightest SUSY particle is uncolored and uncharged. In the GMSB scenario the LSP is always the gravitino, so this condition is automatically fulfilled. Within the mSUGRA and mAMSB scenario, the LSP is required to be the neutralino. Parameter sets that result in a different LSP are excluded.

- We do not impose further restrictions arising from $\mathrm{BR}(b \rightarrow s \gamma)$ 78 and $g_{\mu}-$ 2 [79], which could lead to additional constraints on the three soft SUSY-breaking scenarios. Restrictions of this kind depend on the experimental errors of these observables and the uncertainties in their theoretical prediction and could change considerably if the experimental central values change in the future. Moreover, slight modifications of the SUSY-breaking scenarios which would have only a minor impact on the phenomenology of the models discussed here could have a strong influence on constraints from $\mathrm{BR}(b \rightarrow s \gamma)$ and $g_{\mu}-2$. This could happen, for instance, in the case of $\mathrm{BR}(b \rightarrow s \gamma)$ via the presence of small flavor mixing terms in the SUSY Lagrangian.

As a conservative approach, we therefore do not discard parameter sets which do not fulfill the constraints from $\mathrm{BR}(b \rightarrow s \gamma)$ [78 and $g_{\mu}-2$. It should be noted, however, that if these constraints are imposed and the AMSB scenario is taken at face value, i.e. without any additional contributions, the parameter space allowed by the experimental values of $\operatorname{BR}(b \rightarrow s \gamma)$ and $g_{\mu}-2$ is rather restricted. As for the mSUGRA scenario, the effect of $\mathrm{BR}(b \rightarrow s \gamma)$ would disallow a region with small $M_{1 / 2}$ for large $\tan \beta$, whereas the effect of $g_{\mu}-2$ would be to set an upper bound on the combination of $M_{0}$ and $M_{1 / 2}$, see e.g. Ref. [81]. 
- In the same spirit, we also do not apply any further cosmological constraints, i.e. we do not demand a relic density in the region favored by dark matter constraints, see Ref. [84] and references therein. As in the case of $\mathrm{BR}(b \rightarrow s \gamma)$ and $g_{\mu}-2$, slight modifications of the scenario which do not concern collider phenomenology could have a strong impact on the bounds derived from cosmology. In the case where the LSP relic abundance in the scenarios discussed here is too small to explain the observed amount of cold dark matter (CDM), a further mechanism could provide the additionally required amount of CDM (this would certainly apply to the case of mGMSB). If on the other hand the amount of CDM appears to be too large in a given scenario, "thermal inflation" [85] could offer a mechanism for bringing the CDM density into agreement with the cosmological bounds. Furthermore, the neutralino could turn out to be the NLSP and decay (outside of collider detectors) into a very weakly interacting LSP (e.g. the axino [86]), or there could be a small amount of $R$-parity violation present in the model.

\section{$3 \quad$ Numerical analyses}

\subsection{Experimental bounds from the MSSM Higgs sector}

The results from the Higgs search at LEP have excluded a considerable part of the MSSM parameter space [34]. On the other hand, an excess at about the $3 \sigma$ level has been observed which is compatible with the production of a SM Higgs boson with a mass of about $115 \mathrm{GeV}$ [2]. For our numerical analysis we will focus on three different cases implying different restrictions on the MSSM parameter space. In case (I) we investigate the full parameter space which is allowed in the three scenarios when taking into account the exclusion bounds from the Higgs search and the further constraints discussed in the previous section. In case (II) and case (III), on the other hand, we specifically focus on the interpretation of the excess observed in the Higgs search at LEP as production of the lightest $\mathcal{C} \mathcal{P}$-even Higgs boson of the MSSM (case (II)) and of the heavier $\mathcal{C} \mathcal{P}$-even Higgs boson of the MSSM (case (III)).

(I) The results of the search for the MSSM Higgs bosons are usually interpreted in three different benchmark scenarios [15]. The 95\% C.L. exclusion limit for the SM Higgs boson of $m_{H}>113.5 \mathrm{GeV}$ applies also for the lightest $\mathcal{C} \mathcal{P}$-even Higgs boson of the MSSM in the parameter region of large $M_{A}$ and/or small tan $\beta$. In the unconstrained MSSM this bound is reduced to $m_{h}>91.0 \mathrm{GeV} 34$ for $M_{A} \lesssim 150 \mathrm{GeV}$ and $\tan \beta \gtrsim 8$ as a consequence of a reduced coupling of the Higgs to the $\mathrm{Z}$ boson. For the $\mathcal{C P}$-odd Higgs boson a lower bound of $M_{A}>91.9 \mathrm{GeV}$ has been obtained [34. In order to correctly interpolate between the parameter regions where the SM lower bound of $m_{H}>113.5 \mathrm{GeV}$ and the bound $m_{h}>91.0 \mathrm{GeV}$ apply, we use the result for the Higgs-mass exclusion given with respect to the reduced $Z Z h$ coupling squared (i.e. $\sin ^{2}\left(\beta-\alpha_{\text {eff }}\right.$ ), see eq. (16)) [87]. We have compared the excluded region with the theoretical prediction obtained at the two-loop level for $m_{h}$ and $\sin ^{2}\left(\beta-\alpha_{\text {eff }}\right)$ for each parameter set (using $\left.m_{t}=175 \mathrm{GeV}\right)$. 
Another important constraint on $m_{h}$ comes from the searches in $p \bar{p}$ collisions at Run I of the Tevatron 88. No evidence of a signal of the type $p \bar{p} \rightarrow b \bar{b} h \rightarrow b \bar{b} b \bar{b}$ has been found. This leads to an improvement of the LEP limits in the region of large $\tan \beta, \tan \beta \gtrsim 50$. Since the bounds obtained at the Tevatron are given only in the no-mixing and $m_{h}^{\max }$ scenario [15, 88], they do not necessarily apply to all cases of our present analysis. As a conservative treatment, we therefore do not use the Tevatron bound for excluding models in the high $\tan \beta$ region. It should be noted, however, that owing to the REWSB constraint in our analysis below we do not find allowed models for $\tan \beta \gtrsim 60$.

(II) In this scenario the LEP excess is interpreted as production of the lightest $\mathcal{C P}$-even Higgs boson of the MSSM, and we thus focus on the parameter regions in the three soft SUSY-breaking scenarios where

$$
m_{h}=115 \pm 2 \mathrm{GeV} .
$$

The assumed error of $\pm 2 \mathrm{GeV}$ is somewhat larger than the region favored by the LEP data, in order to allow for some theoretical uncertainties from unknown higher order corrections in the Higgs boson mass calculation (note that the dominating theoretical uncertainty is related to the experimental error of the top-quark mass; we focus in our analysis on the value $m_{t}=175 \mathrm{GeV}$, the corresponding $m_{h}$ values for different values of $m_{t}$ can be obtained from the approximate relation $\delta m_{h} / \delta m_{t}=\mathcal{O}(1)$ ). In order to allow an interpretation of the LEP excess in terms of the lightest MSSM Higgs boson, it is furthermore necessary that the production and decay rates of $h$ are similar to those of the SM Higgs boson. We therefore in addition demand $\sin ^{2}\left(\beta-\alpha_{\text {eff }}\right) \gtrsim 0.8$, which results in a production cross section for the Higgs strahlung process, $e^{+} e^{-} \rightarrow Z h \sim \sin ^{2}\left(\beta-\alpha_{\text {eff }}\right)$ close to the SM cross section. Furthermore, we require that the $h b \bar{b}$ coupling in the MSSM is not strongly suppressed compared to the SM case. The $h b \bar{b}$ coupling differs from the corresponding SM coupling in two ways. Firstly, it has an additional factor $\sin \alpha_{\text {eff }} / \cos \beta$ (appearing squared in the branching ratio). We demand that $\sin ^{2} \alpha_{\text {eff }} / \cos ^{2} \beta \gtrsim 0.8$. Secondly, the $h b \bar{b}$ vertex can be affected by gluino loop corrections (and less importantly also gaugino loop corrections) 89, 90]. Usually they are parameterized via $\Delta m_{b}$

$$
\Delta m_{b} \simeq \frac{2 \alpha_{s}}{3 \pi} m_{\tilde{g}} \mu \tan \beta I\left(m_{\tilde{b}_{1}}, m_{\tilde{b}_{2}}, m_{\tilde{g}}\right)+\frac{Y_{t}}{4 \pi} A_{t} \mu \tan \beta I\left(m_{\tilde{t}_{1}}, m_{\tilde{t}_{2}}, \mu\right),
$$

where $Y_{t}=h_{t}^{2} /(4 \pi)$ and

$$
I(a, b, c)=\frac{a^{2} b^{2} \ln \left(a^{2} / b^{2}\right)+b^{2} c^{2} \ln \left(b^{2} / c^{2}\right)+c^{2} a^{2} \ln \left(c^{2} / a^{2}\right)}{\left(a^{2}-b^{2}\right)\left(b^{2}-c^{2}\right)\left(a^{2}-c^{2}\right)} .
$$

The main correction to the $h b \bar{b}$ coupling is proportional to $1 /\left(1+\Delta m_{b}\right)$. In our analysis of case (II) we additionally demand that $\left|\Delta m_{b}\right|<0.5$.

(III) In this scenario we investigate whether the LEP excess can be interpreted as the production of the heavy $\mathcal{C P}$-even Higgs boson in the MSSM. In order to allow this 
interpretation, $H$ has to have SM-like couplings to the $Z$, i.e. $\cos ^{2}\left(\beta-\alpha_{\text {eff }}\right) \gtrsim 0.8$. In this case the $h$ production via Higgs strahlung, $e^{+} e^{-} \rightarrow Z h$, is highly suppressed, whereas the associated production $e^{+} e^{-} \rightarrow A h$ could be beyond the kinematic reach of LEP. We apply a bound of $m_{h}+M_{A}>206 \mathrm{GeV}$ in this scenario. It should be noted that this bound is very conservative, since values of $m_{h}+M_{A}$ as low as about $190 \mathrm{GeV}$ are not excluded from the Higgs search at LEP [34. As in case (II) we also require that the decay of the heavy $\mathcal{C} \mathcal{P}$-even Higgs boson is $\mathrm{SM}$ like, i.e. the dominating decay channel is $H \rightarrow b \bar{b}$. Therefore we demand $\cos ^{2} \alpha_{\text {eff }} / \cos ^{2} \beta>0.8$.

\section{2 mSUGRA}

For the numerical analysis we have scanned over about 50000 models, where the parameters have been randomly chosen in the intervals

$$
\begin{aligned}
50 \mathrm{GeV} & \leq M_{0} \leq 1 \mathrm{TeV} \\
50 \mathrm{GeV} & \leq M_{1 / 2} \leq 1 \mathrm{TeV}, \\
-3 \mathrm{TeV} \leq A_{0} & \leq 3 \mathrm{TeV}, \\
1.5 \leq \tan \beta & \leq 60, \\
& \operatorname{sign} \mu= \pm 1 .
\end{aligned}
$$

Although we have scanned over about 50000 models, we show in the figures of this paper a subset of around 5000 (randomly) selected points to keep the density of the points at a reasonable level. This has been done for all three soft SUSY-breaking scenarios. No reduction of the data points is applied for the cases (I)-(III) in parameter regions with a small density of points, i.e. in particular for $m_{h}<113 \mathrm{GeV}$ and $\sin ^{2}\left(\beta-\alpha_{\text {eff }}\right)<0.99$.

We first analyze the allowed parameter region in the Higgs sector of the mSUGRA scenario. In Fig. 1 we show the variation of the light Higgs boson mass with respect to $\tan \beta$ for the three cases defined in Sect. 3.1. Fig. 2 shows the allowed parameter space in the $M_{A}-\tan \beta$ plane. Case (I), corresponding to the models that have passed all experimental and theoretical constraints, is indicated in the figures by big green (light shaded) points. Big red (dark shaded) points indicate case (II), i.e. the subset of case (I) in which $h$ has SM like couplings and its mass lies within $113 \mathrm{GeV} \leq m_{h} \leq 117 \mathrm{GeV}$. The points corresponding to case (III), for which an interpretation of the LEP excess in terms of production of the heavier $\mathcal{C} \mathcal{P}$-even Higgs boson is possible, are displayed as blue stars (indicated by arrows in the plots). The little black dots indicate parameter points which, while in principle possible in the mSUGRA scenario, are rejected because of the experimental and theoretical constraints discussed above.

As a general feature, Fig. 1 shows that $m_{h}$ sharply increases with $\tan \beta$ in the region of low $\tan \beta$, while for $\tan \beta \gtrsim 10$ the $m_{h}$ values saturate. Values of $\tan \beta \gtrsim 60$ are not allowed due to the REWSB constraint. The LEP2 Higgs boson searches exclude the models with $m_{h} \lesssim 113 \mathrm{GeV}$ and $\tan \beta \lesssim 50$. This is contrary to the general LEP2 Higgs boson searches in the $m_{h}^{\max }$ scenario [15, 34, 73], where the exclusion bound on the SM Higgs boson mass applies to $m_{h}$ only for $\tan \beta \lesssim 8$. For larger values of $\tan \beta$ and small $M_{A}$ in the unconstrained MSSM a suppression of the $h Z Z$ coupling is possible, giving rise to a reduced production rate compared to the SM case. In the mSUGRA scenario 


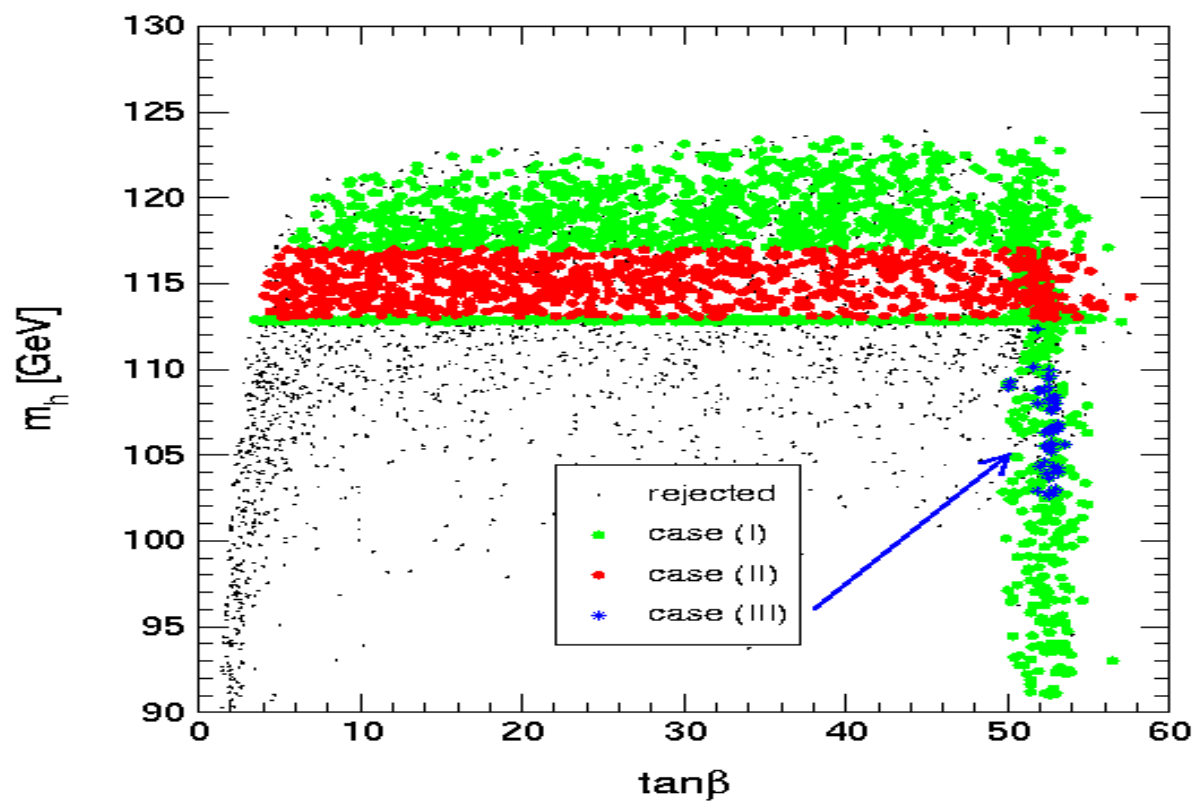

Figure 1: The light $\mathcal{C P}$-even Higgs boson mass $m_{h}$ as a function of $\tan \beta$ in the mSUGRA scenario. The three cases as discussed in Sect. 3.1 are displayed together with the rejected models. Case (I) corresponds to the models that have passed all theoretical and experimental constraints. Case (II) is the subset of case (I) with $m_{h}$ values in the region favored by recent LEP Higgs searches, $113 \mathrm{GeV} \leq m_{h} \leq 117 \mathrm{GeV}$, and SM like couplings of the $h$. In case (III), whose parameter points are indicated by an arrow for better readability, the heavier $\mathcal{C} \mathcal{P}$-even Higgs boson lies in the region $113 \mathrm{GeV} \leq m_{H} \leq 117 \mathrm{GeV}$, while the lighter one has a suppressed coupling to the $Z$ boson and is too heavy to be produced in associated production.

a significant suppression of $\sin ^{2}\left(\beta-\alpha_{\text {eff }}\right)$ (i.e. the $h Z Z$ coupling) occurs only for a small allowed parameter region with $\tan \beta \gtrsim 50$, see Fig. 3 . This feature can be understood from the correlation between $M_{A}$ and $\tan \beta$ shown in Fig. 2. Small values of $M_{A}$ with $M_{A} \lesssim 150 \mathrm{GeV}$, which are necessary for values of $\sin ^{2}\left(\beta-\alpha_{\text {eff }}\right) \ll 1$, are only possible for $\tan \beta \gtrsim 50$. For $\tan \beta \lesssim 45$ we find that $M_{A}$ is always larger than about $300 \mathrm{GeV}$, giving thus rise to a SM like behavior of the $h Z Z$ coupling.

As one can see in Figs. [1, 21 case (III) can indeed be realized in the mSUGRA scenario in a small parameter region where $50 \lesssim \tan \beta \lesssim 55,103 \mathrm{GeV} \lesssim m_{h}, M_{A} \lesssim 113 \mathrm{GeV}$ and $m_{H}=115 \pm 2 \mathrm{GeV}$. It should be noted, however, that this parameter region is close to the exclusion bounds obtained at Run I of the Tevatron [88] (which, as discussed above, we have not imposed in the present analysis). With the upcoming results from Run II of the Tevatron it should be possible to fully cover the parameter space compatible with case (III).

From Fig. [ one can read off an upper bound] on the light $\mathcal{C P}$-even Higgs boson mass

7 This bound is $\sim 3 \mathrm{GeV}$ lower than the one obtained in Ref. 26] due to additional constraints imposed in the present analysis, see Sect. 2.6. 


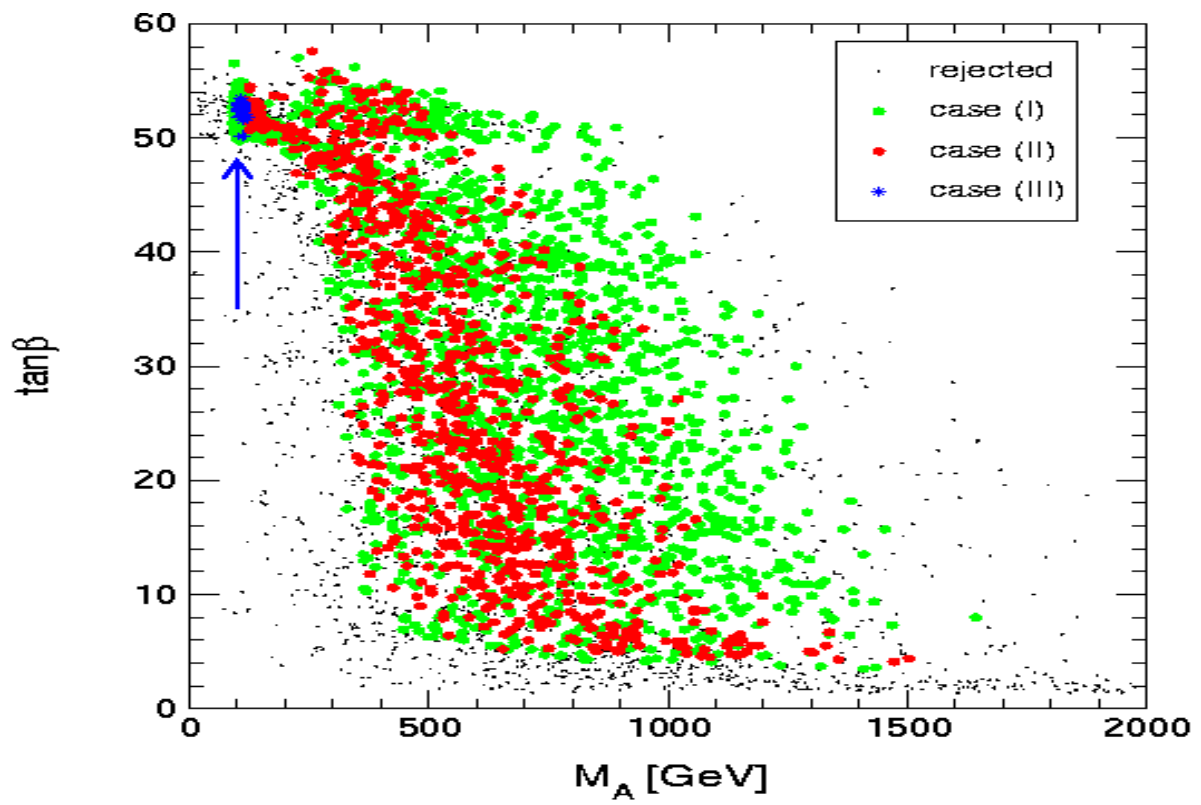

Figure 2: Allowed parameter space within the mSUGRA scenario in the $M_{A}-\tan \beta$ plane for the three cases defined in Sect. 3.1.

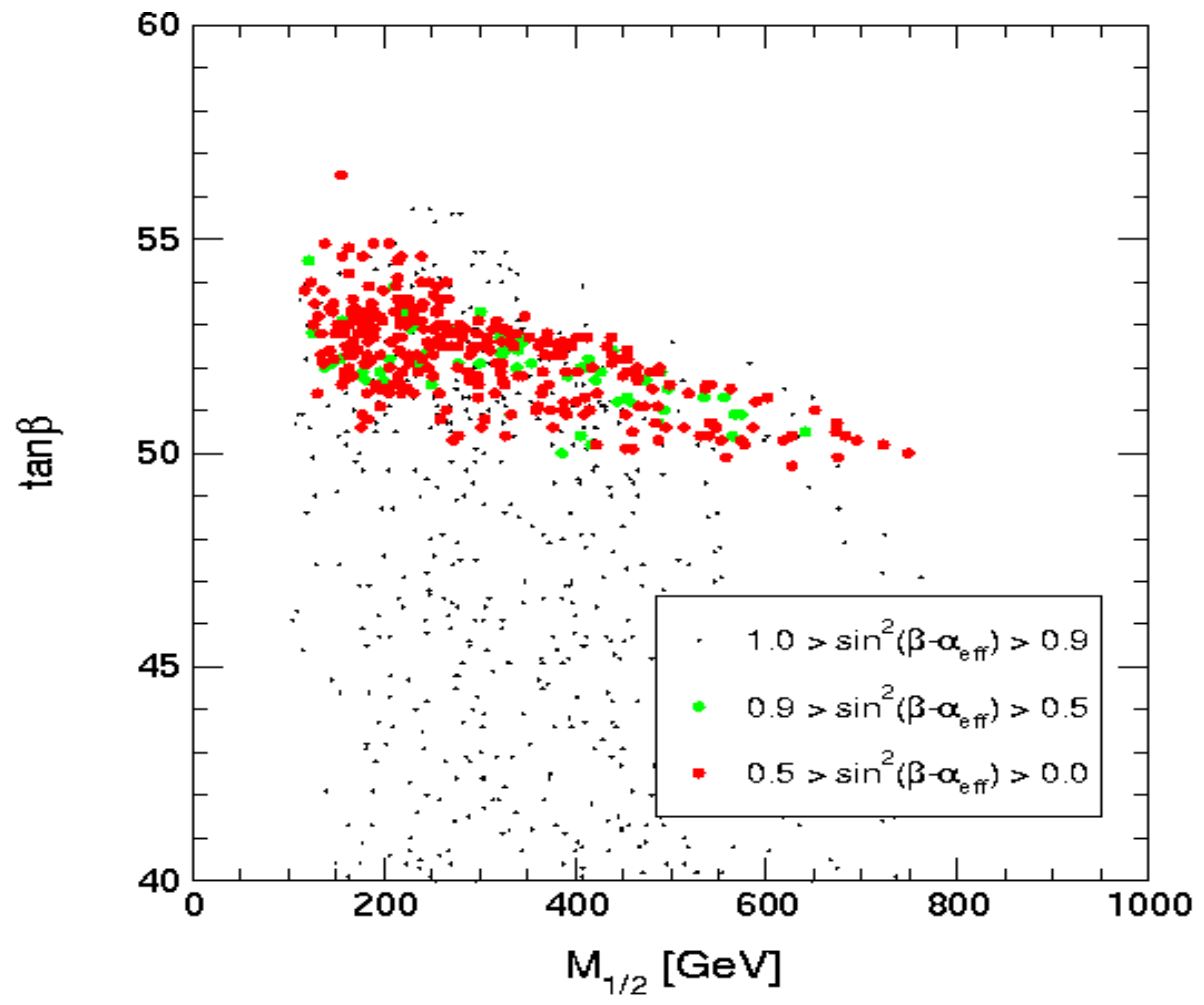

Figure 3: The values for $\sin ^{2}\left(\beta-\alpha_{\text {eff }}\right)$ realized in the mSUGRA scenario are given in the $M_{1 / 2}-\tan \beta$ plane. 


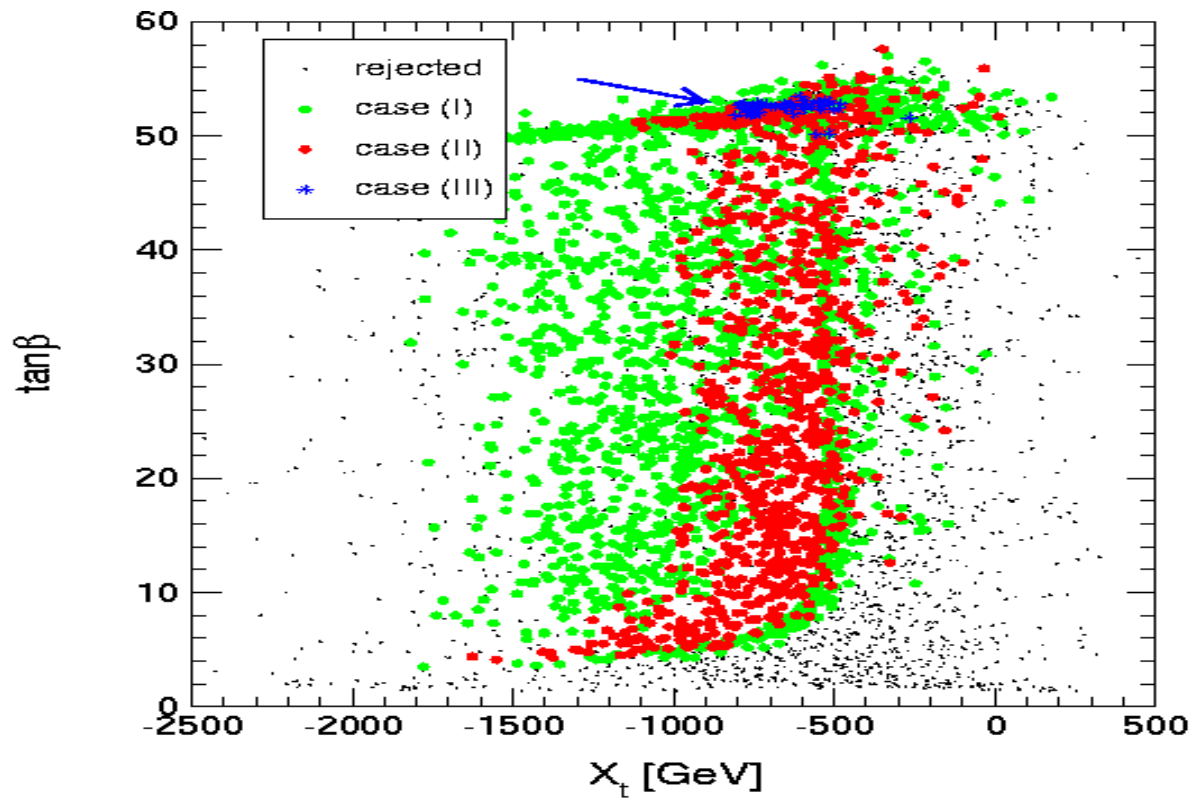

Figure 4: Allowed parameter space within the mSUGRA scenario in the plane of the mixing parameter in the $\tilde{t}$ sector, $X_{t}$, and $\tan \beta$ for the three cases defined in Sect. 3.1.

in the mSUGRA scenario of

$$
m_{h}^{\max } \lesssim 124 \mathrm{GeV} \quad(\mathrm{mSUGRA})
$$

Values close to this upper limit on $m_{h}$ are reached in a large region of moderate and large values of $\tan \beta, 20 \lesssim \tan \beta \lesssim 50$.

A lower bound on $\tan \beta$ is inferred in the mSUGRA scenario,

$$
\tan \beta \gtrsim 3.3 \quad(\mathrm{mSUGRA})
$$

It should be noted that the two bounds quoted here refer to $m_{t}=175 \mathrm{GeV}$.

The upper bound on $m_{h}$ is about $6 \mathrm{GeV}$ lower than the one in the unconstrained MSSM [4, 34], and the limit on $\tan \beta$ is also more restrictive. This is caused by the fact that not all parameter combinations of the unconstrained MSSM can be realized in the mSUGRA scenario. In order to obtain the largest values for $m_{h}$ in particular large values of the parameter $X_{t}$,

$$
X_{t} \equiv A_{t}-\mu / \tan \beta
$$

are necessary, which appears in the off-diagonal element of the $\tilde{t}$ mass matrix. Nonlogarithmic genuine two-loop contributions to $m_{h}$ give rise to an asymmetry with respect to the sign of $X_{t}$, and the maximum value obtained for $m_{h}$ is about $5 \mathrm{GeV}$ higher for $X_{t}>0$ than for $X_{t}<0$ [4, 91].

In Fig. \# the allowed parameter space in the $X_{t}-\tan \beta$ plane of the mSUGRA scenario is depicted for the three cases discussed above. The figure shows that the mSUGRA scenario strongly favors negative values of $X_{t}$. The absence of models with large positive 


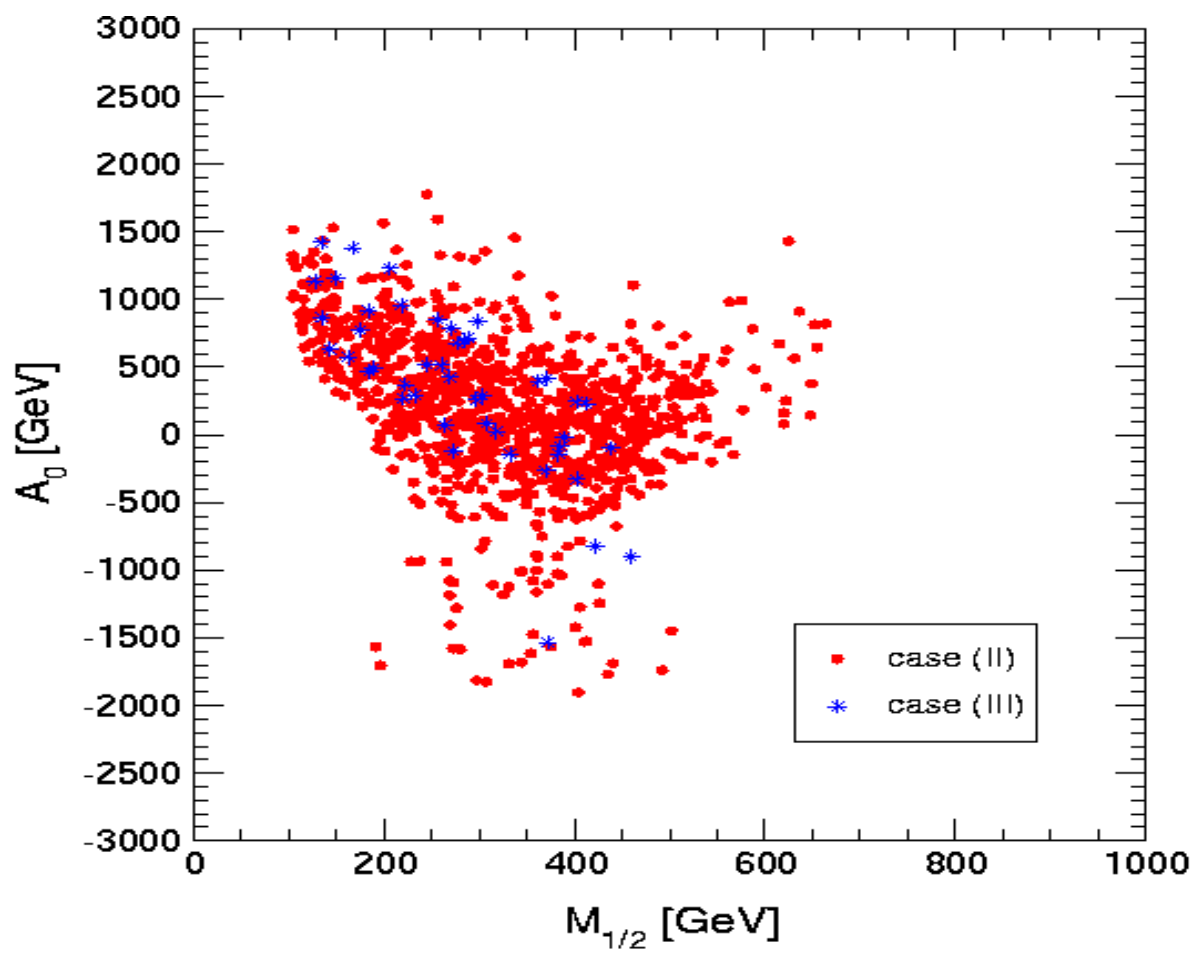

Figure 5: Cases (II) and (III) in the mSUGRA scenario are shown in the $M_{1 / 2}-A_{0}$ plane.

$X_{t}$ is the main reason for the decrease in the upper bound within the mSUGRA compared to the unconstrained MSSM.

Concerning the lower bound on $m_{h}$, we find the same bound as in the unconstrained MSSM, i.e. $m_{h} \gtrsim 91 \mathrm{GeV}$. As discussed above, $m_{h}$ values below $113 \mathrm{GeV}$ being compatible with the LEP exclusion bounds are only possible in a small parameter region with $\tan \beta \gtrsim 50$ in the mSUGRA scenario.

We now turn to the restrictions on the parameter space of the underlying mSUGRA parameters, $M_{0}, M_{1 / 2}$ and $A_{0}$, which are obtained if the LEP excess is interpreted as a signal, i.e. for the cases (II) and (III). Figs. 5, 6 show that the cases (II) and (III) result in similar allowed regions of parameter space. While for $M_{0}$ the whole range up to $1 \mathrm{TeV}$ is allowed, $M_{1 / 2}$ is restricted to $M_{1 / 2} \lesssim 650 \mathrm{GeV}$, see Fig. 5. In Fig. 6 the cases (II) and (III) are shown in the $M_{0}-A_{0}$ plane. $\left|A_{0}\right|$ is restricted to $\left|A_{0}\right| \lesssim 2 M_{0}$. For the special case $A_{0}=0$ we find that $M_{0}$ is bounded from above by $M_{0} \lesssim 700 \mathrm{GeV}$.

We find that $M_{1 / 2}$ values as low as $100 \mathrm{GeV}$ (these values are only obtained for not too small $M_{0}, M_{0} \gtrsim 300 \mathrm{GeV}$; the lower bound on $M_{1 / 2}$ for smaller values of $M_{0}$ is about $M_{1 / 2} \gtrsim 200 \mathrm{GeV}$ ) are compatible with the interpretation of the LEP excess as a signal of the lightest $\mathcal{C P}$-even Higgs boson in the MSSM. This result is in contrast to the analysis in Ref. [92], where for $m_{t}=175 \mathrm{GeV}$ and $m_{h} \geq 113 \mathrm{GeV}$ a lower bound of $M_{1 / 2} \gtrsim 310 \mathrm{GeV}$ has been found for $A_{0}=0$, while we obtain a lower bound of $M_{1 / 2} \gtrsim 200 \mathrm{GeV}$, see Fig. 5 . The main difference between our result and the one obtained in Ref. [92] can be traced to the inclusion of genuine non-logarithmic two-loop corrections in the result for $m_{h}$ in the 


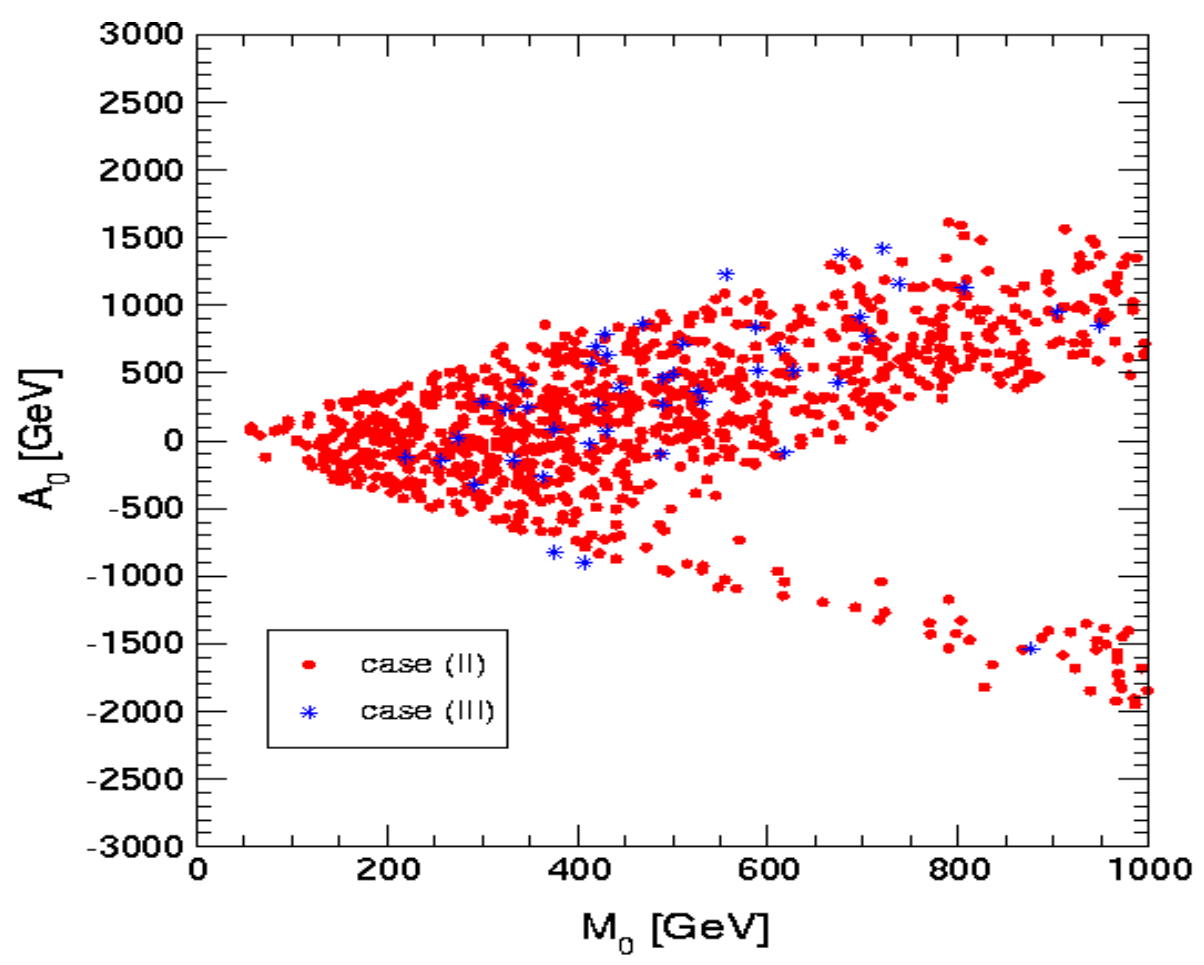

Figure 6: Cases (II) and (III) in the mSUGRA scenario are shown in the $M_{0}-A_{0}$ plane.

present paper (see also Ref. [81]). The lower values found for $M_{1 / 2}$ in the present analysis give rise to a different low energy spectrum for the SUSY particles. As an exemplary case, we find that $M_{0} \approx M_{1 / 2} \approx 200 \mathrm{GeV}$ and $-400 \mathrm{GeV} \lesssim A_{0} \lesssim 400 \mathrm{GeV}$ is compatible with $m_{h}=115 \pm 2 \mathrm{GeV}$ together with all the constraints listed in Sect. 2.6.3. In this example the heaviest SUSY particle is the gluino with a mass of $\sim 590 \mathrm{GeV}$ and all the scalar quarks have masses about $500 \mathrm{GeV}$ or lower, depending on $A_{0}$ and on $\tan \beta$. The SUSY spectrum in the three soft SUSY-breaking scenarios will be analyzed in more detail in Sect. 3.5.

Finally, we investigate the $h b \bar{b}$ coupling in the mSUGRA scenario in comparison with the SM case, where the decay into $b$ quarks is the dominant decay channel of the Higgs boson. The $h b \bar{b}$ coupling is mainly altered in two ways compared to the SM: it has an extra factor $\sin \alpha_{\text {eff }} / \cos \beta$ and it receives a correction $\sim 1 /\left(1+\Delta m_{b}\right)$, see eq. (20).

Fig. 7 shows the different values of $\sin ^{2} \alpha_{\text {eff }} / \cos ^{2} \beta$ (which enters the $h \rightarrow b \bar{b}$ decay rate) realized within the mSUGRA scenario in the $M_{1 / 2}-\tan \beta$ plane. The figure shows that a significant enhancement of the $h b \bar{b}$ coupling is possible over a wide range of the mSUGRA parameter space. In these parameter regions the sensitivity in the Higgs search via the $h \rightarrow b \bar{b}$ channel is in general slightly increased compared to the SM case. On the other hand, the increase in the $h \rightarrow b \bar{b}$ partial width leads in general to a reduced branching ratio of $h \rightarrow \gamma \gamma$, making thus the search via this channel at the LHC more difficult 81$]]^{8}$.

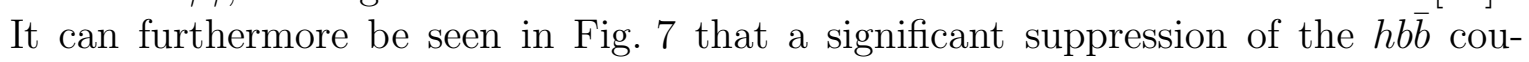
pling is only possible in a small fraction of the mSUGRA parameter space. Values of

\footnotetext{
${ }^{8}$ For a similar analysis for the charged Higgs bosons, see Ref. [94].
} 


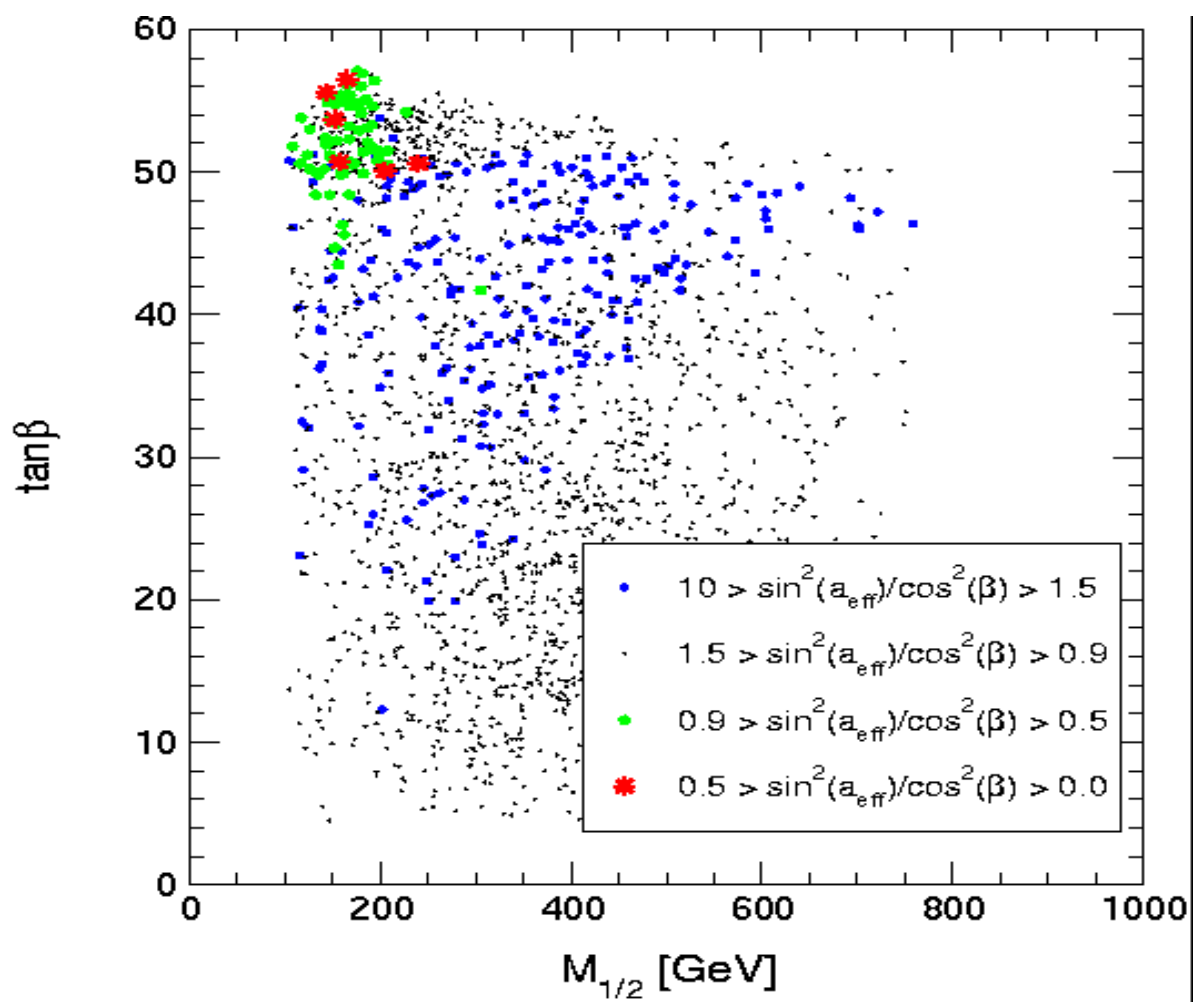

Figure 7: The values for $\sin ^{2} \alpha_{\text {eff }} / \cos ^{2} \beta$ realized in the mSUGRA scenario are given in the $M_{1 / 2}-\tan \beta$ plane.

$\sin ^{2} \alpha_{\text {eff }} / \cos ^{2} \beta<0.7$ are only obtained in the parameter region $\tan \beta \gtrsim 50$.

In Fig. 8 the quantity $\Delta m_{b}$ is analyzed in the mSUGRA scenario. The maximal values obtained for $\Delta m_{b}$ in the mSUGRA scenario are about \pm 0.4 (where values of $\left|\Delta m_{b}\right|>0.3$ are only realized for $\tan \beta \gtrsim 40)$. The figure shows that values of $\sin ^{2}\left(\beta-\alpha_{\text {eff }}\right) \ll 1$ (corresponding to a suppressed coupling of the Higgs to vector bosons and lower allowed values for $m_{h}$ ) are always correlated in the mSUGRA scenario with negative values of $\Delta m_{b}$, giving rise to an enhancement of the $h b \bar{b}$ coupling. Positive values for $\Delta m_{b}$ (corresponding to a suppression of the $h b \bar{b}$ coupling) are only possible if the Higgs boson couples with full strength to $W$ and $Z$.

\section{3 mGMSB}

For this paper, about 40000 mGMSB models were generated under well defined hypotheses described in Sect. 2.3, using the program SUSYFIRE [55] and adopting the phenomenological approach of Refs. [51 53], see also Ref. [27]. Concerning the variation of the highenergy parameters one should keep in mind that the lower bound on $\Lambda$ arises from the requirement that $m_{\mathrm{NLSP}} \geq 100 \mathrm{GeV}$, while its upper bound as well as the upper bound on $M_{\text {mess }}$ originate mainly from the upper bound imposed on SUSY particle masses, see Sect. 2.6.3. The upper bounds on $\Lambda$ and $M_{\text {mess }}$ automatically restrict $N_{\text {mess }}$ from above, as explained in Sect. 2.3. The above restrictions yield the following variations of the 


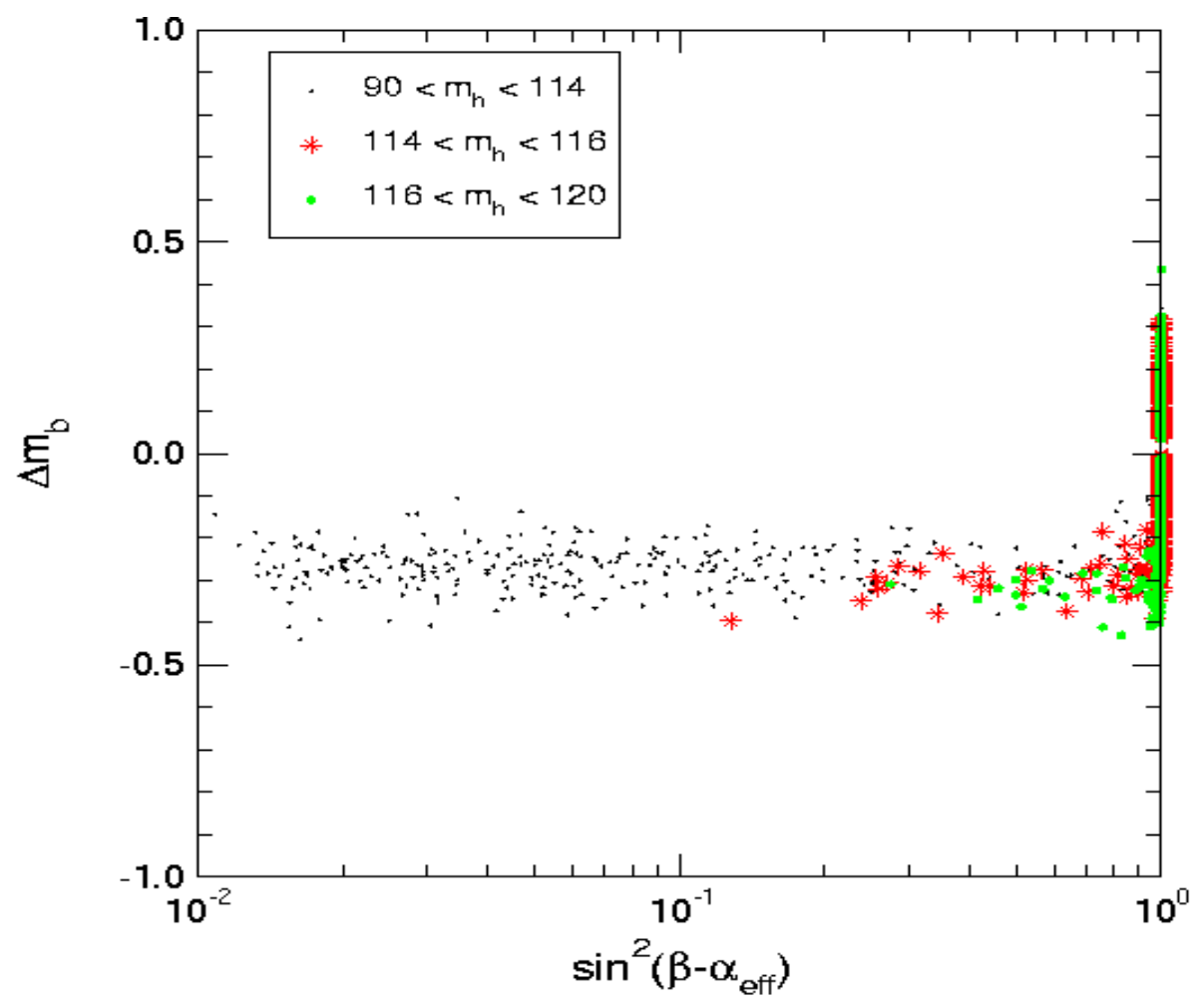

Figure 8: The allowed values in the mSUGRA scenario for $\sin ^{2}\left(\beta-\alpha_{\text {eff }}\right)$ and the quantity $\Delta m_{b}$ (see eq. (20)). The corresponding values for $m_{h}$ are also indicated.

high-energy parameters:

$$
\begin{aligned}
10^{4} \mathrm{GeV} & \leq \Lambda \leq 2 \times 10^{5} \mathrm{GeV} \\
1.01 \Lambda & \leq M_{\text {mess }} \leq 10^{5} \Lambda \\
1 \leq N_{\text {mess }} & \leq 8 \\
1.5 & \leq \tan \beta \leq 55 \\
& \operatorname{sign} \mu= \pm 1 .
\end{aligned}
$$

As above, we first analyze the allowed parameter region in the Higgs sector in this scenario. In Fig. 9 we show the variation of the light Higgs boson mass with respect to $\tan \beta$ for case (I) and case (II) defined in Sect. 3.1. Case (III), where the LEP excess is interpreted as a signal of the heavier $\mathcal{C} \mathcal{P}$-even Higgs boson in the MSSM, is not realized in the mGMSB scenario. Fig. 10 shows the allowed parameter space in the $M_{A}-\tan \beta$ plane.

It can be seen from the two figures that the experimental and theoretical constraints discussed in Sect. 2.6 have a bigger effect on the parameter space than in the case of the mSUGRA scenario. In particular, they significantly influence the upper bound on $m_{h}$, which is reduced in this way by about $3 \mathrm{GeV}$. Like in the mSUGRA case, the LEP2 Higgs boson searches exclude the models with $m_{h} \lesssim 113 \mathrm{GeV}$ and $\tan \beta \lesssim 50$. A significant suppression of $\sin ^{2}\left(\beta-\alpha_{\text {eff }}\right)$ (i.e. the $h Z Z$ coupling) occurs only for a small allowed 


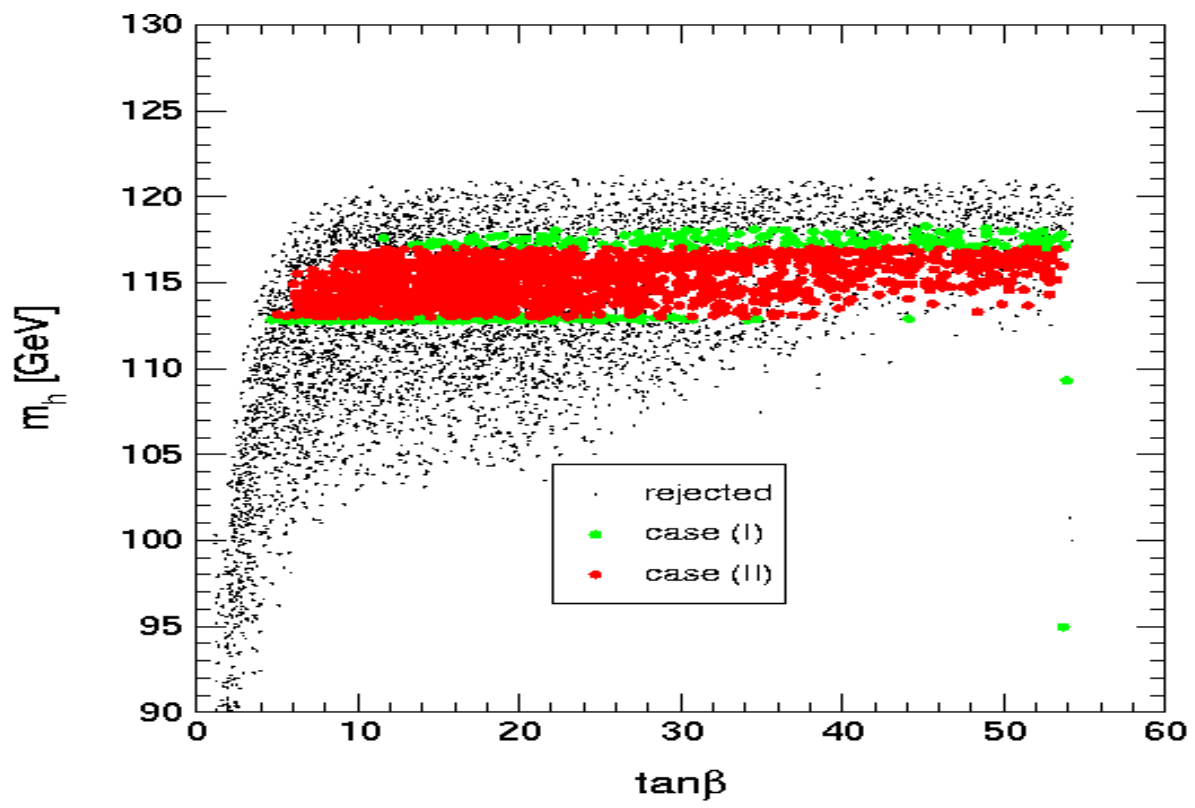

Figure 9: The light Higgs boson mass $m_{h}$ as a function of $\tan \beta$ in the mGMSB scenario. Case (I) and case (II) as discussed in Sect. 3.1 are displayed together with the rejected models. Case (I) corresponds to the models that have passed all theoretical and experimental constraints. Case (II) is the subset of case (I) with $m_{h}$ values in the region favored by recent LEP Higgs searches, $113 \mathrm{GeV} \leq m_{h} \leq 117 \mathrm{GeV}$, and SM like couplings of the $h$. Case (III), where the LEP excess is interpreted as a signal of the heavier $\mathcal{C P}$-even Higgs boson in the MSSM, is not realized in the mGMSB scenario.

parameter region with $\tan \beta \gtrsim 50$ (the lower density of points with $\tan \beta \gtrsim 50$ and $m_{h}<113 \mathrm{GeV}$ as compared to Fig. 11 has no direct physical meaning; it is mainly due to the fact that $\tan \beta$ has been varied on a logarithmic scale in the mGMSB scenario, while a linear scale has been chosen in the mSUGRA scenario). This can be understood from Fig. 10, which shows that values of $M_{A} \lesssim 300 \mathrm{GeV}$ are only realized for $\tan \beta \gtrsim 50$. Values of $\tan \beta \gtrsim 55$ are not allowed due to the REWSB constraint.

For the upper bound on the light $\mathcal{C P}$-even Higgs boson mass in the mGMSB scenario we obtaing

$$
m_{h}^{\max } \lesssim 119 \mathrm{GeV} \quad(\mathrm{mGMSB}) .
$$

Values close to this upper limit on $m_{h}$ are reached in a large region of moderate and large values of $\tan \beta, 20 \lesssim \tan \beta \lesssim 50$.

A lower bound on $\tan \beta$ is inferred in the mGMSB scenario,

$$
\tan \beta \gtrsim 4.6 \quad(\mathrm{mGMSB})
$$

As above, the two bounds quoted here refer to $m_{t}=175 \mathrm{GeV}$.

9 This bound is $\sim 4 \mathrm{GeV}$ lower than the one obtained in Ref. [27, mainly due to additionally imposed constraints like the "naturalness" bound on the scalar quark masses, see Sect. 2.6.3. 


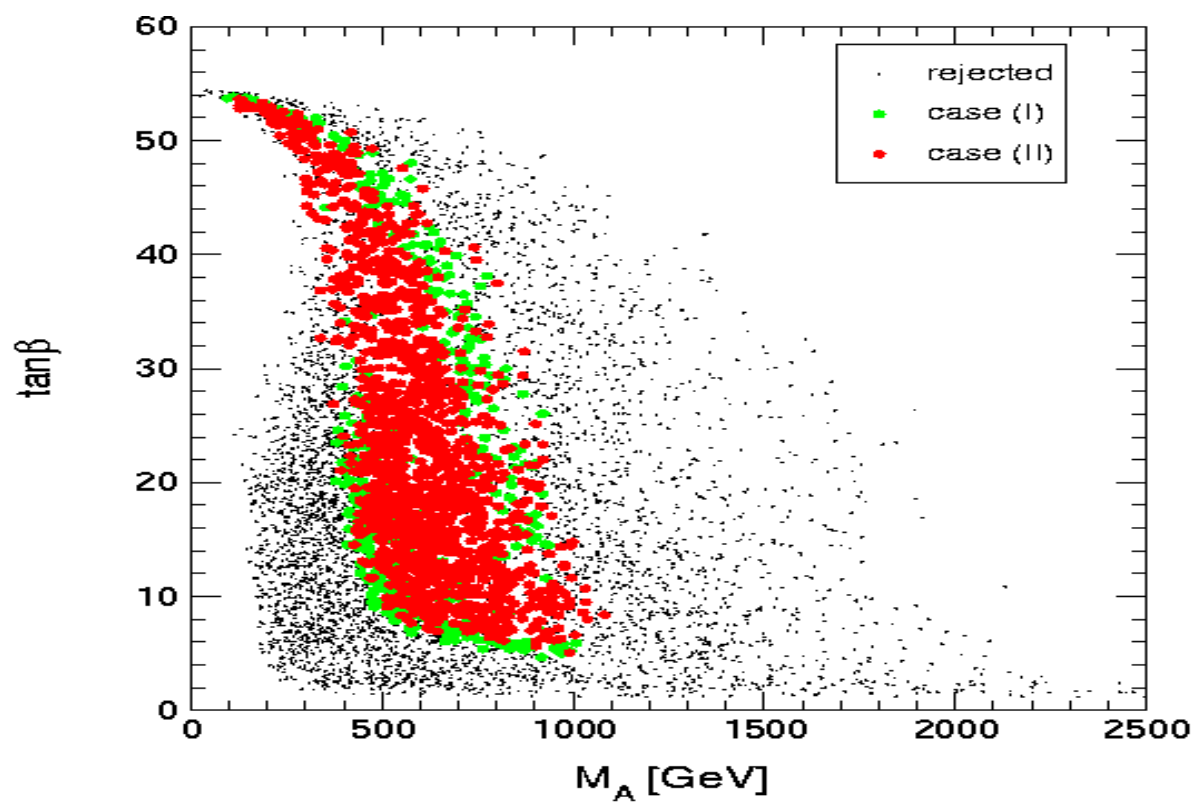

Figure 10: Allowed parameter space within the mGMSB scenario in the $M_{A}-\tan \beta$ plane for case (I) and case (II) defined in Sect. 3.1.

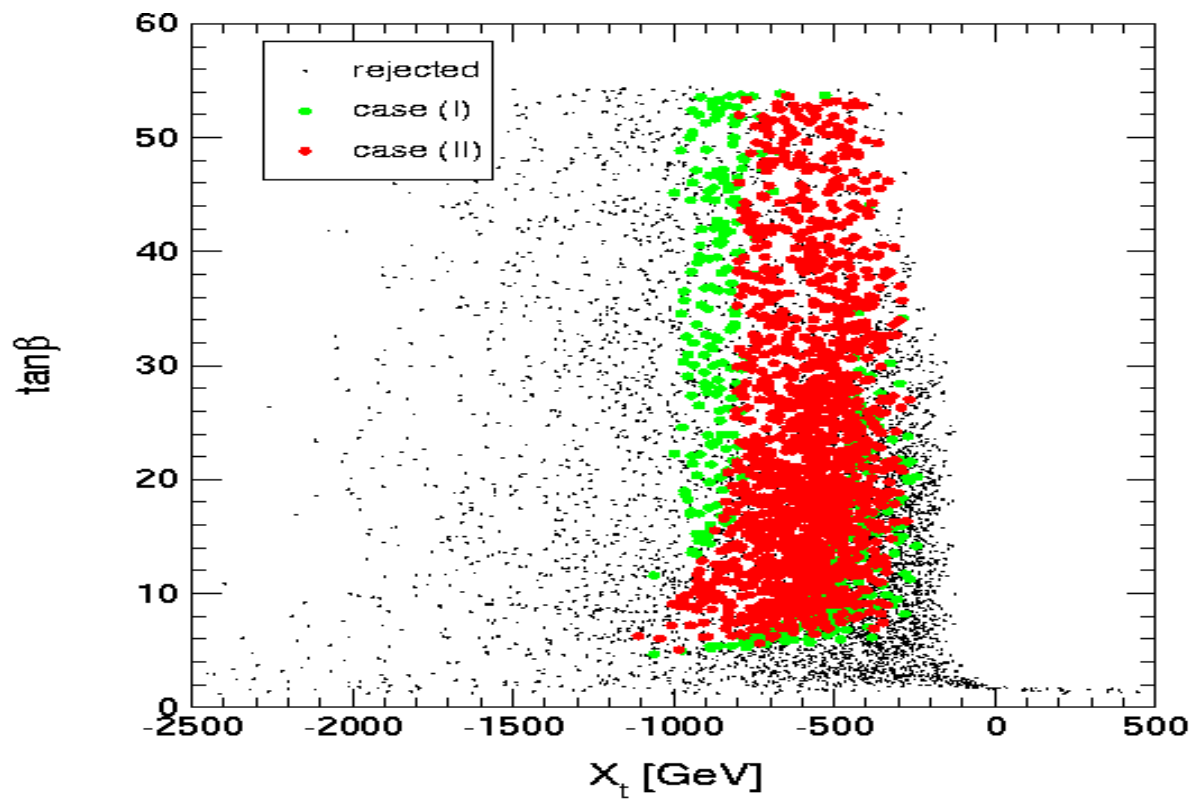

Figure 11: Allowed parameter space within the mGMSB scenario in the plane of the mixing parameter in the $\tilde{t}$ sector, $X_{t}$, and $\tan \beta$ for case (I) and case (II) defined in Sect. 3.1.

As in the mSUGRA scenario, the restrictions on the mixing parameter $X_{t}$ (see eq. (25)) arising from the parameter correlations in the mGMSB scenario (and the upper bound imposed in eq. (18)) are the main effect causing the decrease in the upper bound on $m_{h}$ 


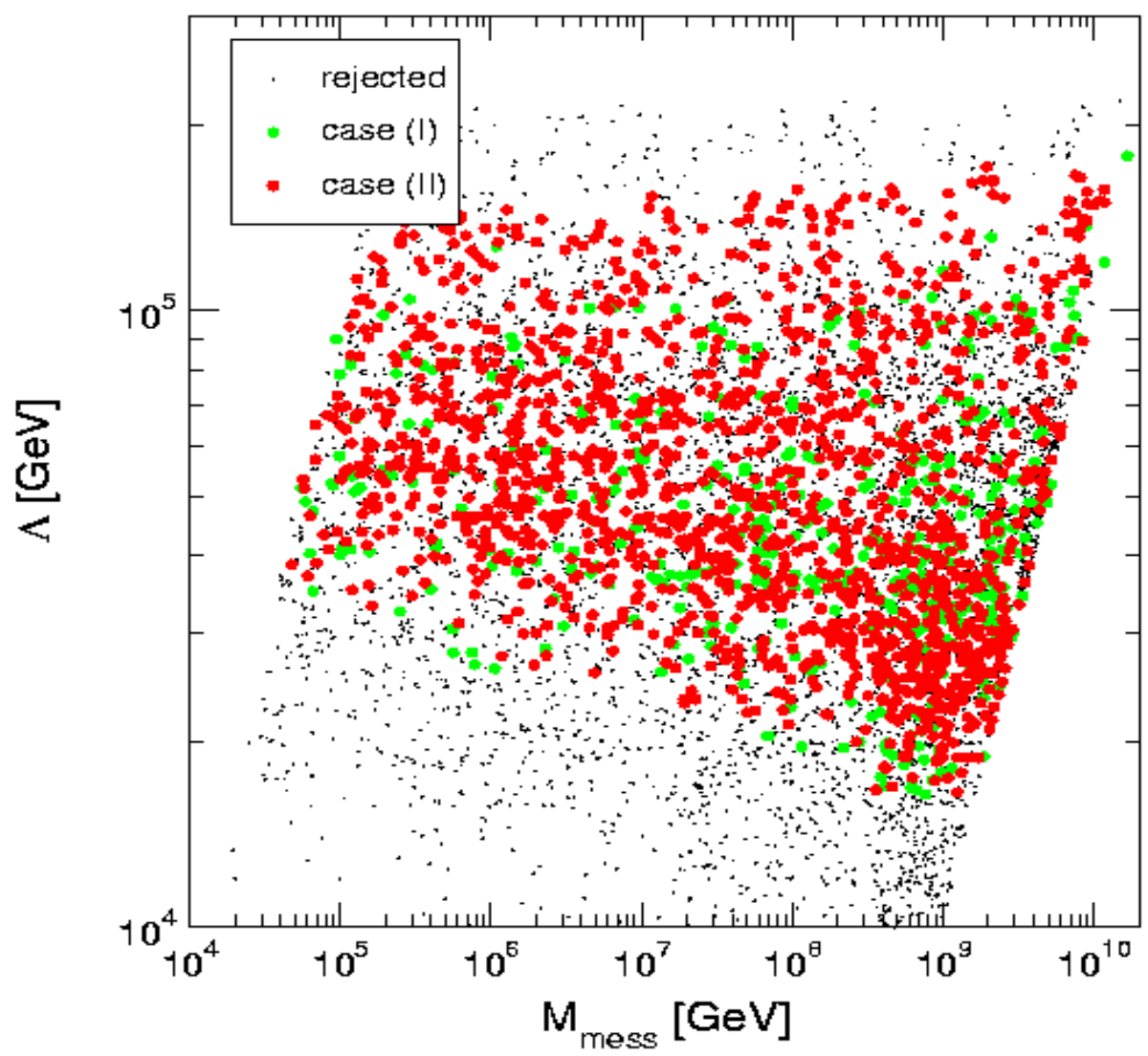

Figure 12: Cases (I) and (II) in the mGMSB scenario are shown in the $M_{\text {mess }}-\Lambda$ plane.
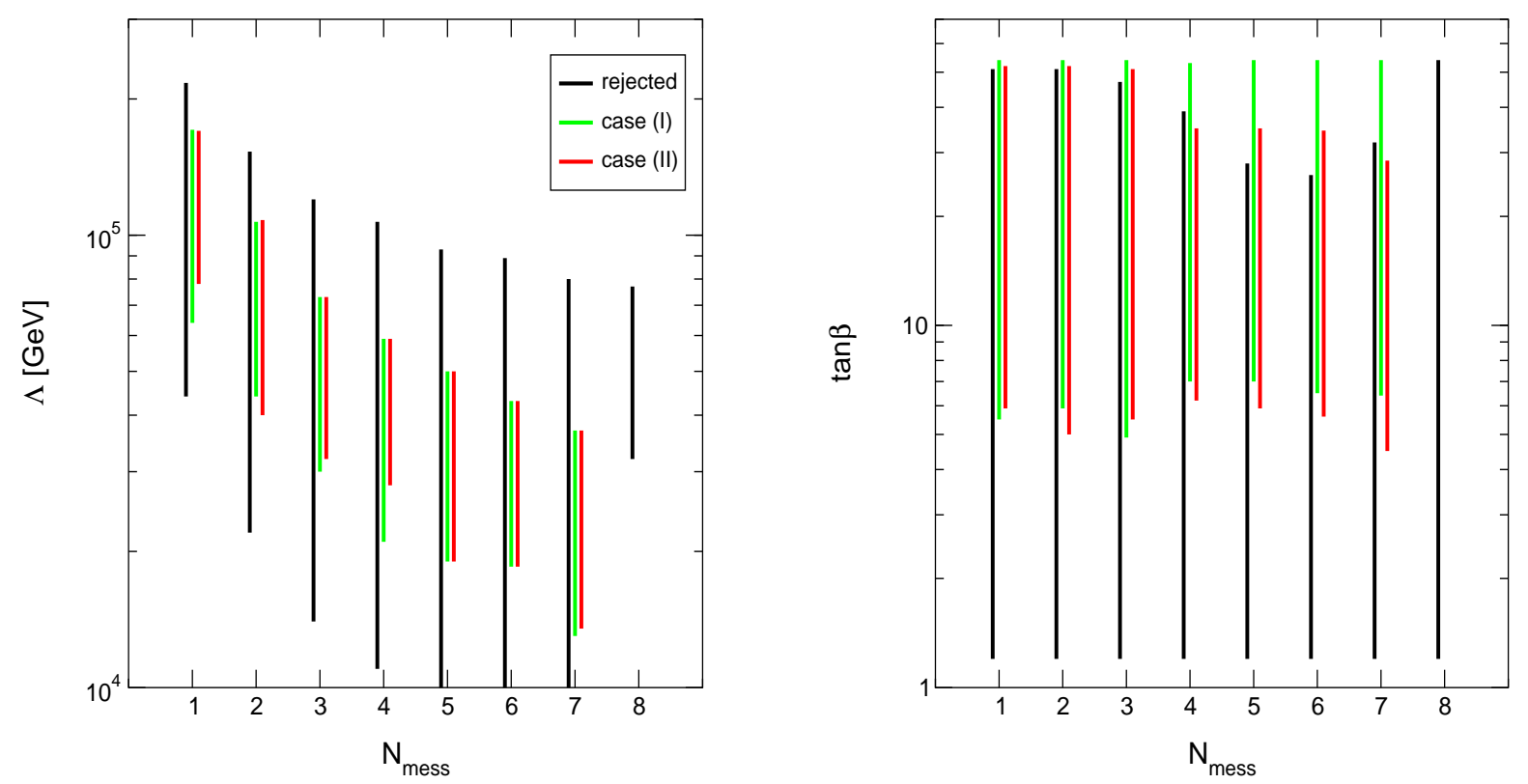

Figure 13: Cases (I) and (II) in the mGMSB scenario are shown in the $N_{\text {mess }}-\Lambda(\tan \beta)$ plane in the left (right) plot. The legend shown in the left plot applies to both plots. 
as compared to the unconstrained MSSM. Fig. 11 shows that only negative values for $X_{t}$ are allowed in the mGMSB scenario. Compared to the mSUGRA case (see Fig. (1) the allowed parameter region for $X_{t}$ is smaller and is furthermore shifted towards smaller values of $\left|X_{t}\right|$.

Concerning the underlying GMSB parameters, $M_{\text {mess }}, N_{\text {mess }}$ and $\Lambda$, no severe restrictions can be deduced for the cases (I) and (II), see Figs. 12, 13. In Fig. 12 we show the allowed regions in the $M_{\text {mess }}-\Lambda$ plane. The experimental and theoretical constraints imposed in our analysis affect in particular the region of low $M_{\text {mess }}$ and low $\Lambda$. In the left plot of Fig. 13 the allowed regions in the $N_{\text {mess }}-\Lambda$ plane are presented. Lower values of $N_{\text {mess }}$ correspond to higher values of $\Lambda$. This is a consequence of the boundary values imposed on the physical masses in eq. (8). The Higgs boson mass constraints cut away a significant part of the $\Lambda$ range for each value of $N_{\text {mess }}$. We only find allowed parameter regions for $N_{\text {mess }} \leq 7$ (although higher values of $N_{\text {mess }}$ might be allowed if the upper bound of $M_{\text {mess }} / \Lambda$ is relaxed.) The right plot of Fig. 13 shows the $N_{\text {mess }}-\tan \beta$ plane. Case (II) corresponds to about the same allowed region as case (I), apart from the values $N_{\text {mess }} \geq 4$, where the highest values of $\tan \beta$ are not allowed in case (II).

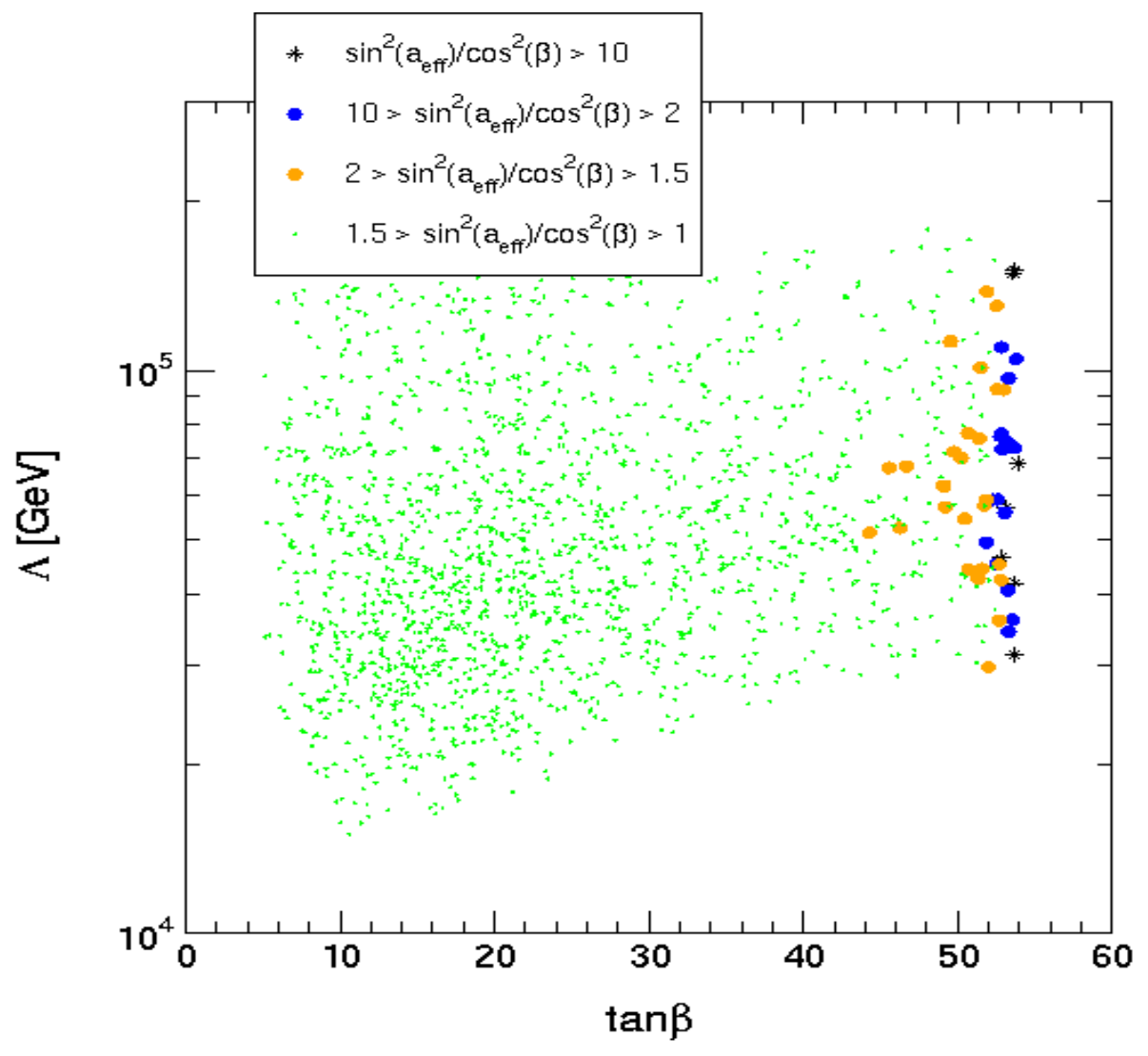

Figure 14: The values for $\sin ^{2} \alpha_{\text {eff }} / \cos ^{2} \beta$ realized in the mGMSB scenario are given in the $\tan \beta-\Lambda$ plane.

We also investigate the $h b \bar{b}$ coupling within the mGMSB scenario. In Fig. 14 the different values of $\sin ^{2} \alpha_{\text {eff }} / \cos ^{2} \beta$ realized within the mGMSB scenario are shown in the 
$\tan \beta-\Lambda$ plane. In contrast to the mSUGRA case, no values of $\sin ^{2} \alpha_{\text {eff }} / \cos ^{2} \beta<1$ exist, i.e. no suppression of the $h b \bar{b}$ coupling occurs in this way. As above, a significant enhancement of the $h b \bar{b}$ coupling is possible. This applies in particular to the region of the highest values of $\tan \beta$.

We have also analyzed the quantity $\Delta m_{b}$ (see eq. (20)) within the mGMSB scenario. The absolute value of $\Delta m_{b}$ is smaller in the mGMSB scenario than in the mSUGRA case and does not exceed $\left|\Delta m_{b}\right|=0.2$. Values of $\left|\Delta m_{b}\right|>0.1$ are only realized for $\tan \beta \gtrsim 35$.

\section{4 mAMSB}

According to the description presented in Sect. 2.4 about 50000 models have been created. The GUT scale parameters have been varied in the ranges

$$
\begin{aligned}
20 \mathrm{TeV} & \leq m_{\mathrm{aux}} \leq 100 \mathrm{TeV} \\
0 \leq m_{0} & \leq 2 \mathrm{TeV} \\
1.5 \leq \tan \beta & \leq 60 \\
\operatorname{sign} \mu & = \pm 1
\end{aligned}
$$

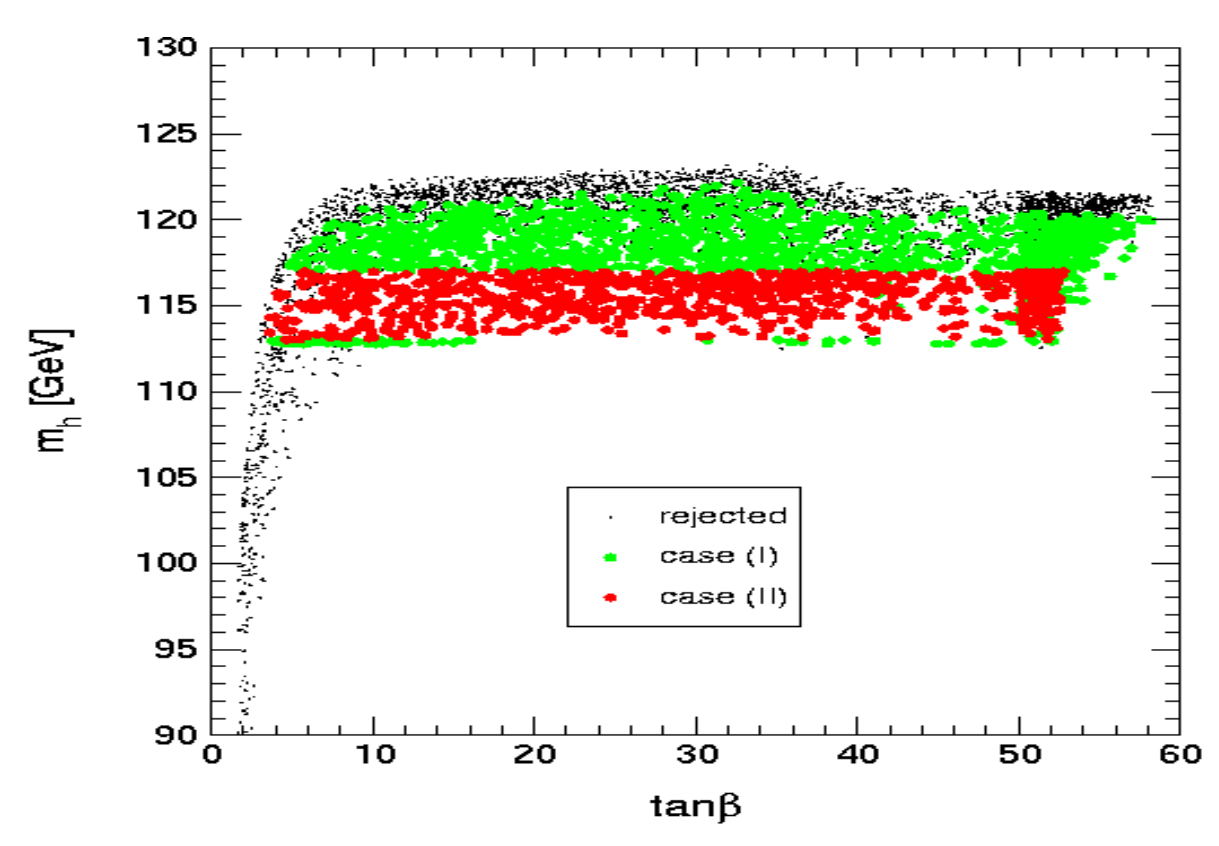

Figure 15: The light $\mathcal{C P}$-even Higgs boson mass $m_{h}$ as a function of $\tan \beta$ in the mAMSB scenario. Case (I) and case (II) as discussed in Sect. 3.1 are displayed together with the rejected models. Case (I) corresponds to the models that have passed all theoretical and experimental constraints. Case (II) is the subset of case (I) with $m_{h}$ values in the region favored by recent LEP Higgs searches, $113 \mathrm{GeV} \leq m_{h} \leq 117 \mathrm{GeV}$, and SM like couplings of the $h$. Case (III), where the LEP excess is interpreted as a signal of the heavier $\mathcal{C P}$-even Higgs boson in the MSSM, is not realized in the mAMSB scenario. 


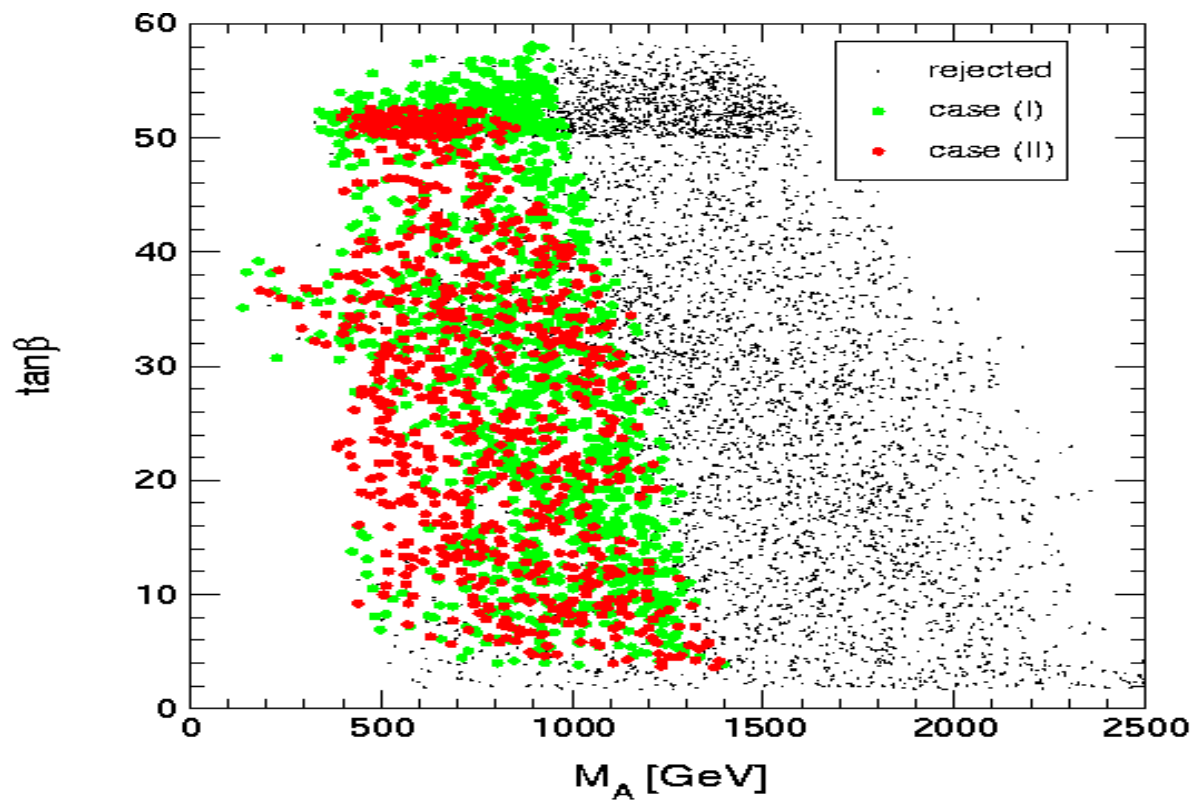

Figure 16: Allowed parameter space within the mAMSB scenario scenario in the $M_{A}-$ $\tan \beta$ plane for case (I) and case (II) defined in Sect. 3.1.

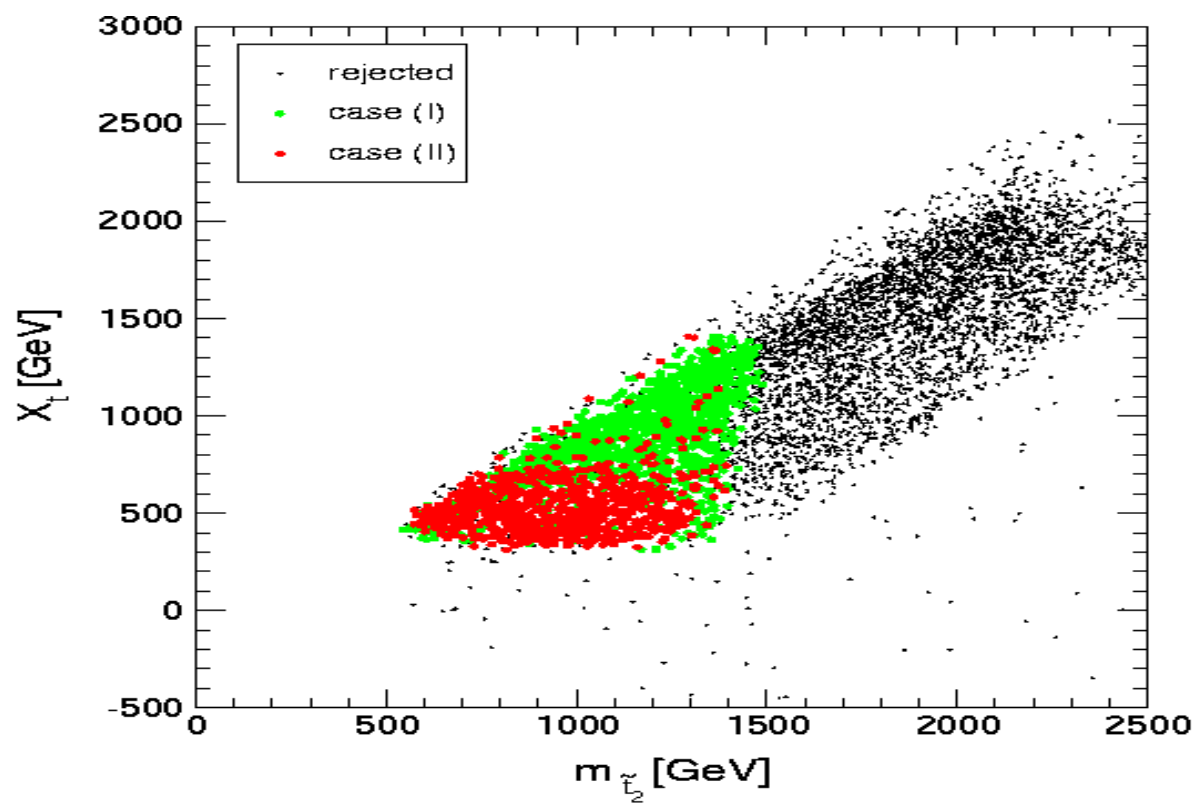

Figure 17: Allowed parameter space within the mAMSB scenario in the plane of the heavier scalar top mass, $m_{\tilde{t}_{2}}$, and the mixing parameter in the $\tilde{t}$ sector, $X_{t}$, for case (I) and case (II) defined in Sect. 3.1.

The general behavior of the Higgs boson sector has already been described in Ref. 25]. Both $m_{h}$ and $M_{A}$ increase with $m_{\text {aux }}$, which determines the SUSY mass scale. $m_{h}$ depends only very weakly on $m_{0}$ and $\tan \beta$ except for large $m_{0}$ and small $\tan \beta$. $M_{A}$ gets larger 
for larger $m_{0}$ and $\tan \beta$, although the dependence on $\tan \beta$ is rather weak.

In Fig. 15 we show the variation of the light Higgs boson mass with respect to $\tan \beta$ for case (I) and case (II) defined in Sect. 3.1. Case (III), where the LEP excess is interpreted as a signal of the heavier $\mathcal{C} \mathcal{P}$-even Higgs boson in the MSSM, is not realized in the mAMSB scenario (as will be explained below). Fig. 16 shows the allowed parameter space in the $M_{A}-\tan \beta$ plane.

We find the highest values for $m_{h}$ at $\tan \beta$ values of about 35. The experimental and theoretical constraints discussed in Sect. 2.6 reduce the upper bound on $m_{h}$ by $1-2 \mathrm{GeV}$. In the mAMSB scenario we do not find a significant suppression of $\sin ^{2}\left(\beta-\alpha_{\text {eff }}\right)$ (i.e. the $h Z Z$ coupling). As can be seen in Fig. 16, values of $M_{A}$ below $300 \mathrm{GeV}$ are only realized in the interval $30 \leq \tan \beta \leq 40$, and $M_{A}$ stays always above about $150 \mathrm{GeV}$, giving thus rise to a SM like $h Z Z$ coupling. As a consequence, the LEP2 Higgs boson searches exclude all models with $m_{h} \lesssim 113 \mathrm{GeV}$. This affects mainly the region $\tan \beta \lesssim 10$, while for larger values of $\tan \beta$ hardly any mAMSB model results in a Higgs boson mass lower than $113 \mathrm{GeV}$, see Fig. 15. Values larger than $\tan \beta>60$ are not allowed due to the REWSB constraint.

Fig. 16 furthermore shows that the experimental and theoretical constraints discussed in Sect. 2.6 exclude a significant fraction of the parameter space in the $M_{A}-\tan \beta$ plane. In general larger values of $M_{A}$ correspond to smaller $\tan \beta$ (giving rise to a smaller bottom Yukawa coupling $y_{b}$ ) because $m_{H_{d}}^{2}$, which contributes mainly to $M_{A}^{2}$, is larger at low energies due to the RGE running. For larger $\tan \beta$, smaller $M_{A}$ cannot be realized. Otherwise the corresponding $m_{0}$ would be too small to avoid negative slepton masses. The relatively large values required for $M_{A}$ in the mAMSB scenario, on the other hand, exclude the possibility of case (III).

The upper bound on the light $\mathcal{C} \mathcal{P}$-even Higgs boson mass in the mAMSB scenario is (for $m_{t}=175 \mathrm{GeV}$ )

$$
m_{h}^{\max } \lesssim 122 \mathrm{GeV} \quad(\mathrm{mAMSB}) .
$$

Values close to this upper limit on $m_{h}$ are reached in a large region of moderate and large values of $\tan \beta, \tan \beta \gtrsim 10$.

A lower bound on $\tan \beta$ is inferred in the mAMSB scenario (for $m_{t}=175 \mathrm{GeV}$ ),

$$
\tan \beta \gtrsim 3.2 \quad(\mathrm{mAMSB})
$$

As above, we have analyzed the allowed values of the mixing parameter in the scalar top sector, $X_{t}$. Fig. 17 shows the allowed parameter space in the plane of the heavier $\tilde{t}$ mass, $m_{\tilde{t}_{2}}$, and $X_{t}$. In contrast to the mSUGRA and the mGMSB scenarios positive values for $X_{t}$ are preferred. The experimental and theoretical constraints discussed in Sect. 2.6 are seen to have a significant effect, limiting the allowed values of $X_{t}$ to $X_{t} \lesssim 1.5 \mathrm{TeV}$. Fig. 17 shows that in the mAMSB scenario $X_{t}$ is bounded from above, $X_{t} \lesssim m_{\tilde{t}_{2}}$. This is the main reason for the decrease in the upper bound on $m_{h}$ compared to the unconstrained MSSM, since the highest values for $m_{h}$ are reached for values of $X_{t}$ significantly larger than the heavier $\tilde{t}$ mass (see e.g. Refs. [4, 91]).

In Fig. 18 we present the allowed regions in the plane of the high energy input parameters $m_{0}$ and $m_{\text {aux }}$. The experimental and theoretical constraints imposed in our analysis affect in particular the region of large $m_{0}$ and $m_{\text {aux }}$. We find no allowed models 


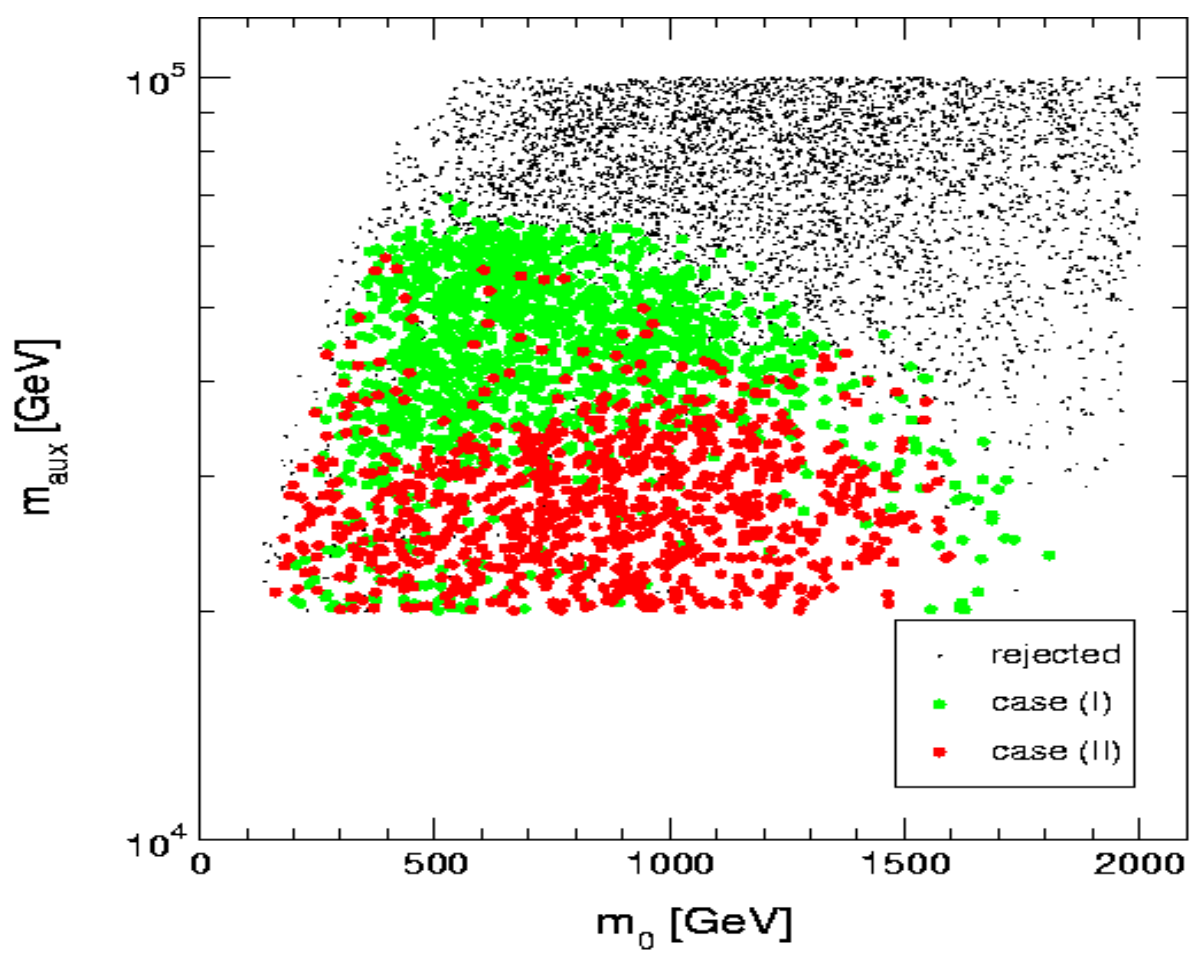

Figure 18: Cases (I) and (II) in the mAMSB scenario are shown in the $m_{0}-m_{\text {aux }}$ plane.

with $m_{\text {aux }} \gtrsim 70 \mathrm{TeV}$. On the other hand, even the smallest values of $m_{0}$ giving rise to acceptable slepton masses lead to allowed parameter points in case (I) and case (II).

Concerning the $h b \bar{b}$ coupling within the mAMSB scenario, we have analyzed the possible values for $\sin ^{2} \alpha_{\text {eff }} / \cos ^{2} \beta$ and $\Delta m_{b}$. We do not find any models with $\sin ^{2} \alpha_{\text {eff }} / \cos ^{2} \beta$ $\lesssim 0.9$ (values of $\sin ^{2} \alpha_{\text {eff }} / \cos ^{2} \beta<1$ only occur for $\tan \beta \gtrsim 40$ ), i.e. the SUSY contributions entering via $\alpha_{\text {eff }}$ do not give rise to a significant reduction of the $h \rightarrow b \bar{b}$ decay rate in the mAMSB scenario. Values of $\sin ^{2} \alpha_{\text {eff }} / \cos ^{2} \beta>10$ are possible for large $\tan \beta$.

The quantity $\Delta m_{b}$ (see eq. (20)) receives large contributions in the mAMSB scenario, in particular for large $\tan \beta$ and relatively small $m_{0}$. This is shown in Fig. 19, where the different values for $\left|\Delta m_{b}\right|$ are indicated in the $m_{0}-\tan \beta$ plane. We find that positive values of $\Delta m_{b}$, leading to a suppression of the $h b \bar{b}$ coupling, are bounded from above by $\Delta m_{b} \lesssim 0.5$. On the other hand, we obtain negative contributions as large as $\Delta m_{b} \approx-0.8$, giving rise to a strongly enhanced $h b \bar{b}$ Yukawa coupling.

\subsection{The SUSY mass spectra in the three SUSY-breaking sce- narios compatible with a possible Higgs signal at LEP}

We finally compare the mass spectra in the three soft SUSY-breaking scenarios assuming that the LEP excess is due to the production of the $h$ or $H$ boson in the MSSM (cases (II) and (III)) and briefly discuss possible implications for SUSY searches at the next 


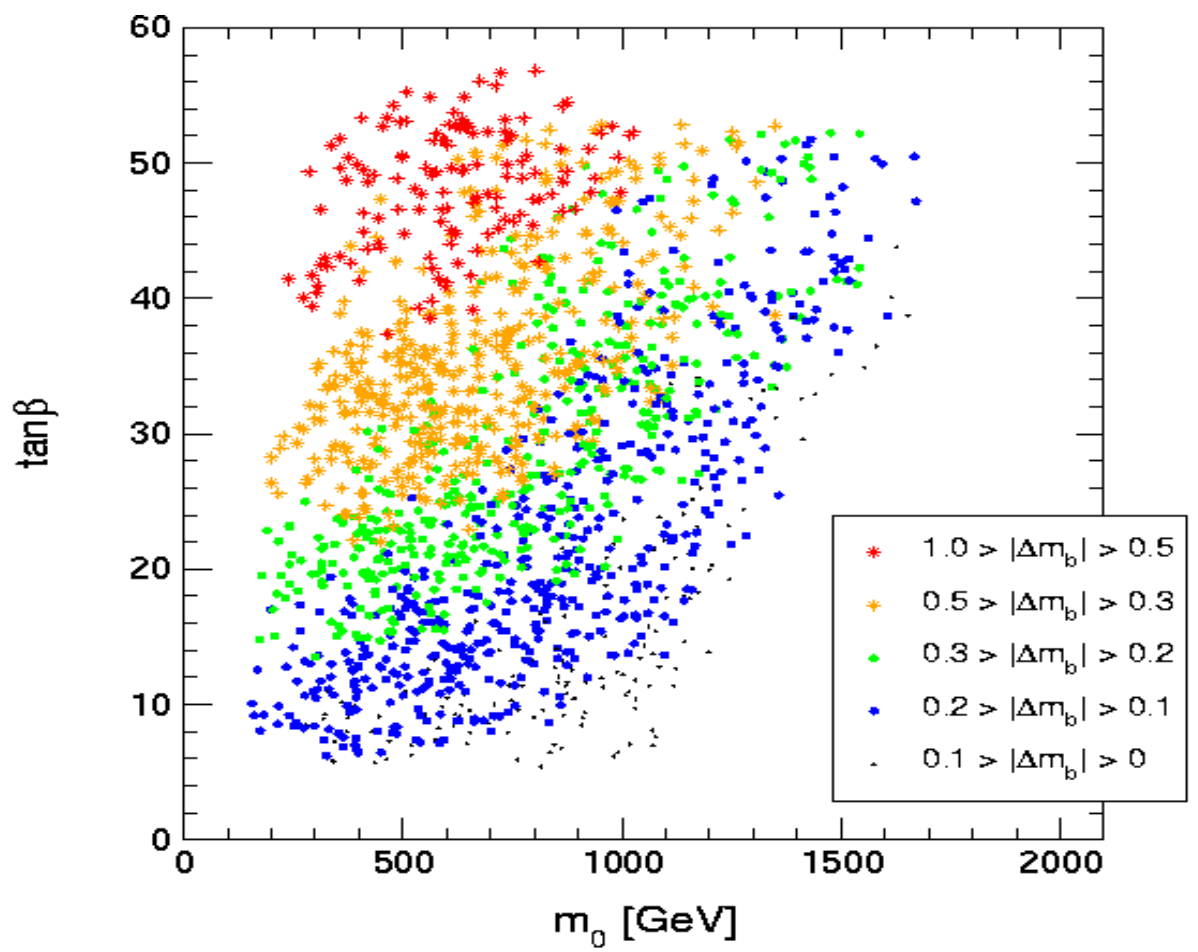

Figure 19: The allowed values for the quantity $\left|\Delta m_{b}\right|$ (see eq. (20)) for different values of $m_{0}$ and $\tan \beta$ in the mAMSB scenario.

generation of colliders喵. In Figs. 20, 21 we show the spectra of the lightest neutralinos, the charginos, the scalar top and bottom quarks, the scalar $\tau$ leptons and of the gluino in the mSUGRA, mGMSB and mAMSB scenarios. The points shown for the mGMSB and mAMSB scenarios correspond to case (II), while for the mSUGRA models we do not distinguish in these models between cases (II) and (III) (in general case (III) results in about the same mass ranges as case (II)).

For the lightest neutralino, in the mSUGRA and mAMSB scenarios values as low as $m_{\tilde{\chi}_{1}} \approx 50 \mathrm{GeV}$ are compatible with case (II), while in the mGMSB scenario the lower bound of $m_{\tilde{\chi}_{1}} \gtrsim 100 \mathrm{GeV}$ holds in accordance with eq. (17). The upper bounds on $m_{\tilde{\chi}_{1}}$ in Fig. 20 are about 200, 300 and $350 \mathrm{GeV}$ in the mAMSB, mSUGRA and mGMSB scenario, respectively. For $m_{\tilde{\chi}_{2}}$, values as low as about $100 \mathrm{GeV}$ are possible in the mSUGRA scenario, while we find upper bounds between about 550 and $650 \mathrm{GeV}$ in the three scenarios.

The lightest chargino is bounded from above by about $200 \mathrm{GeV}$ in case (II) for the mAMSB scenario, by about $550 \mathrm{GeV}$ in the mSUGRA scenario, and by about $650 \mathrm{GeV}$ in the mGMSB scenario. For $m_{\tilde{\chi}_{2}^{+}}$we find a lower bound of about $250 \mathrm{GeV}$ in the mSUGRA and mAMSB scenarios, while the lower bound in the mGMSB scenario is about $350 \mathrm{GeV}$.

Since within the GMSB scenario the LSP is always the gravitino, detection of the lightest neutralino via $\tilde{\chi}_{1}^{0} \tilde{\chi}_{1}^{0}$ production is possible in this scenario if $\sqrt{F}$ is not too large,

\footnotetext{
${ }^{10}$ Phenomenological differences as well as characteristic signatures at future experiments between the three models have also recently discussed in 93 .
} 

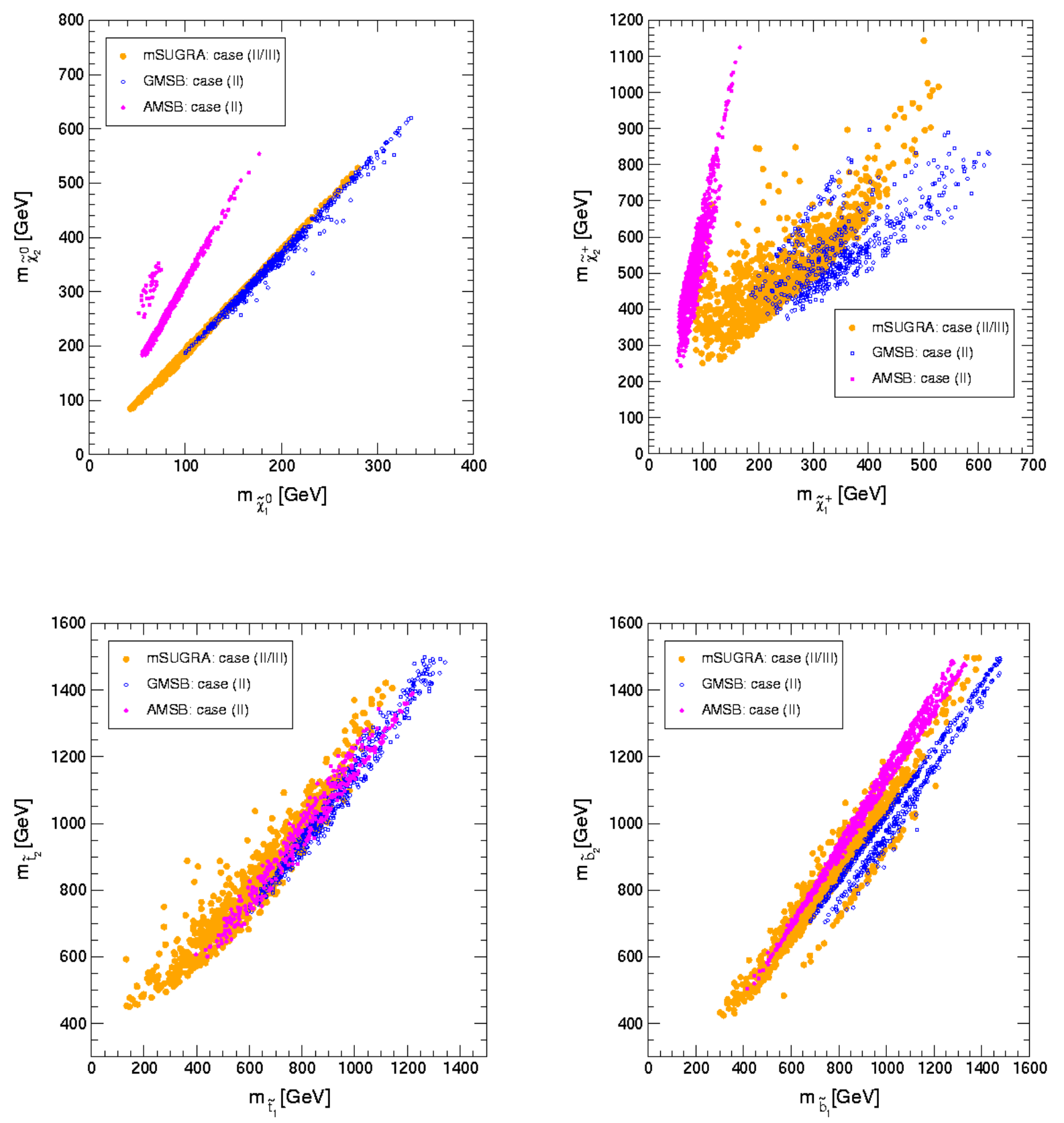

Figure 20: The allowed mass ranges for the lightest neutralinos (upper left plot), the charginos (upper right), the scalar top quarks (lower left) and the scalar bottom quarks (lower right) are shown for the cases (II) and (III) of the mSUGRA, mGMSB and mAMSB scenarios. 

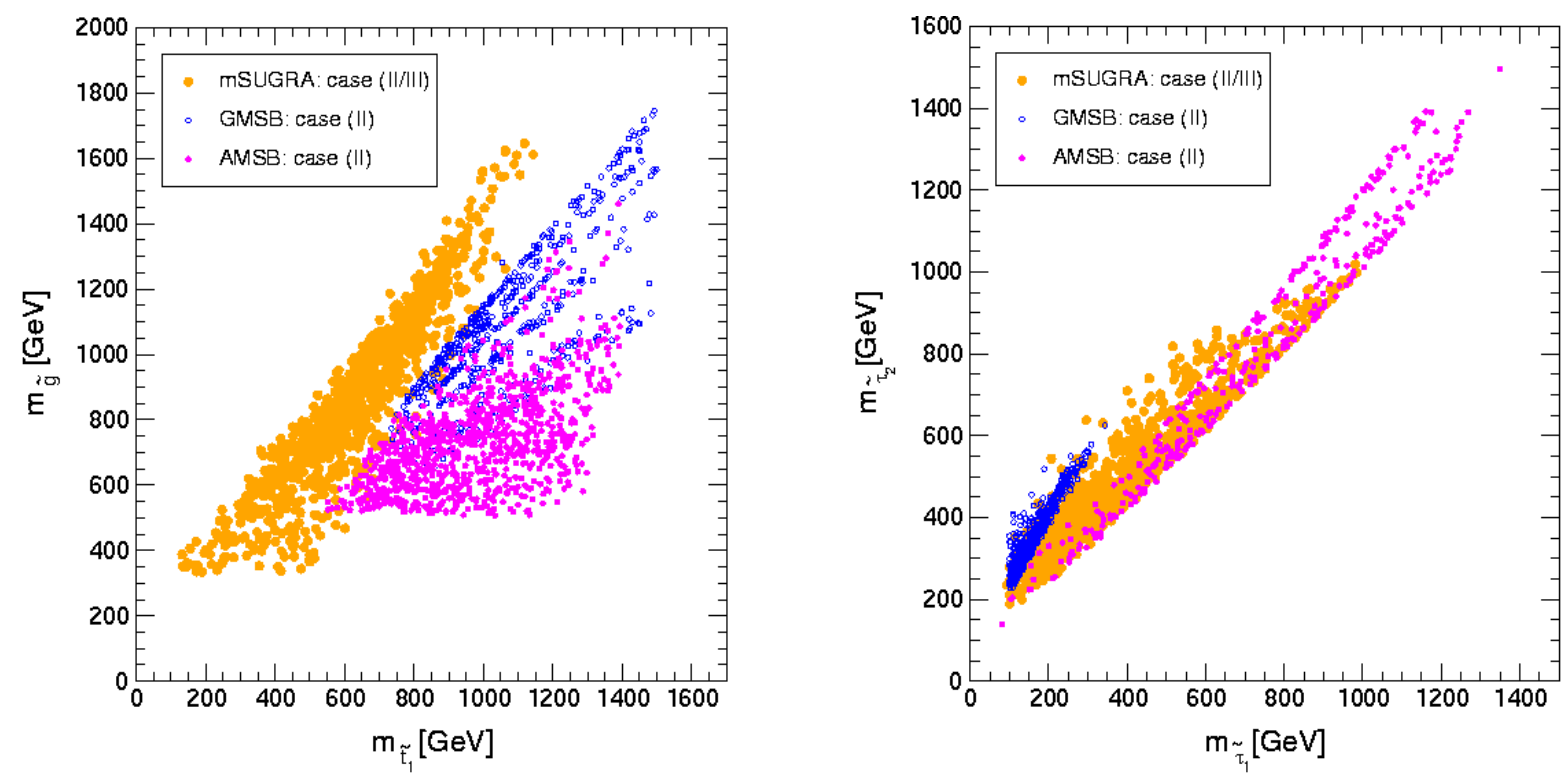

Figure 21: The allowed mass ranges for the gluino (left plot, shown in the $m_{\tilde{t}_{1}}-m_{\tilde{g}}$ plane) and for the scalar $\tau$ leptons (right plot) are shown for the cases (II) and (III) of the mSUGRA, mGMSB and mAMSB scenarios.

while the search in the mSUGRA and mAMSB scenarios has to focus on $\tilde{\chi}_{1}^{0} \tilde{\chi}_{2}^{0}$ associated production.

Within the mSUGRA scenario, the neutralino and chargino searches at Run II of the Tevatron and the LHC will be sensitive to a significant part of the parameter space of the models shown in Fig. 20 95 97. A future $e^{+} e^{-}$linear collider (LC) with a center of mass (CMS) energy of $\sqrt{s} \lesssim 1 \mathrm{TeV}$ will have a very good chance to observe both the associated production of $\tilde{\chi}_{1}^{0} \tilde{\chi}_{2}^{0}$ and the production of the lightest chargino, $\tilde{\chi}_{1}^{+} \tilde{\chi}_{1}^{-}$98, 99.

In the mGMSB scenario the discovery potential at the next generation of colliders for gauginos (and to some extent also for squarks) strongly depends on the lifetime and other properties of the NLSP. The charginos are in general heavier than the two lightest neutralinos, following the mass relation originating from the condition of a unified gaugino mass at the high energy scale, $m_{\tilde{\chi}_{1}^{+}} \approx 2 m_{\tilde{\chi}_{1}^{0}} \approx m_{\tilde{\chi}_{2}^{0}}$. The Tevatron and the LHC will cover at least part of the parameter space in Fig. 20, and the prospects at a LC for neutralino and chargino production in the GMSB scenario are very promising.

A peculiar feature of the mAMSB scenario is that the Wino is always lighter than the Bino [21, 100]:

$$
M_{1}: M_{2}: M_{3} \approx 2.8: 1:-8.3 .
$$

In most of the parameter space, the neutral Wino is the LSP. The NLSP, the charged 
Wino, is generically extremely mass degenerate with the LSP and decays, after its production at a collider, after centimeters into an LSP plus a very soft lepton or pion. The detection of such a charged Wino poses novel experimental challenges since such events escape the conventional triggers. The search for SUSY in the Wino LSP scenario has been studied by several groups [21, 101, 102]. It was pointed out in Ref. [101] that hundreds of Wino pairs can be produced at Run II of the Tevatron with $\sqrt{s}=2 \mathrm{TeV}$ and $\mathcal{L}=2 \mathrm{fb}^{-1}$. Tens of Wino pairs can be produced in association with a jet. The accompanying high energy jet works as a trigger and the detection of 5 events is possible for Wino masses up to $180 \mathrm{GeV}$ at the Tevatron. At an LC with a CMS energy of $\sqrt{s} \lesssim 1 \mathrm{TeV}$ both the associated production of $\tilde{\chi}_{1}^{0} \tilde{\chi}_{2}^{0}$ and of $\tilde{\chi}_{1}^{+} \tilde{\chi}_{1}^{-}$should be observable.

We now turn to the mass spectra of the third generation squarks compatible with cases (II) and (III). Within the mSUGRA scenario the lowest mass values for the third generation squarks are possible, around $150 \mathrm{GeV}$ and $450 \mathrm{GeV}$ for $m_{\tilde{t}_{1}}$ and $m_{\tilde{t}_{2}}$, respectively, and around $300 \mathrm{GeV}$ and $450 \mathrm{GeV}$ for $m_{\tilde{b}_{1}}$ and $m_{\tilde{b}_{2}}$, respectively, see Fig. 20 . Within the GMSB scenario, the low-energy masses generated for colored particles are $\sim \alpha_{s}$, but only $\sim g_{2}$ for uncolored particles. Thus, scalar quarks are in general heavier than sleptons or electroweak gauginos in this scenario. Fig. 20 shows that in the mGMSB and mAMSB scenarios no third generation squark below about $400 \mathrm{GeV}$ is possible in case (II). Similarly, a gluino as light as about $300 \mathrm{GeV}$ is possible in the mSUGRA scenario, while the lower bound on $m_{\tilde{g}}$ is about $200 \mathrm{GeV}$ higher in the mGMSB and mAMSB scenarios, see Fig. 21. As a consequence, the prospects for the production of third generation squarks and the gluino in these scenarios are not very high both at Run II of the Tevatron and a LC with a CMS energy of $\sqrt{s} \lesssim 1 \mathrm{TeV}$. At the LHC, on the other hand, the production of the third generation squarks and the gluino is guaranteed (it should be noted, however, that this conclusion relies on the "naturalness bound" imposed in our analysis, see Sect. 2.6.3).

Finally we analyze the mass spectrum of the scalar $\tau$ leptons in the three scenarios, see Fig. 21. In the mGMSB scenario, stringent upper limits on the scalar $\tau$ masses of

$m_{\tilde{\tau}_{1}} \lesssim 400 \mathrm{GeV}$ and $m_{\tilde{\tau}_{2}} \lesssim 600 \mathrm{GeV}$ apply, giving rise to good prospects for production of scalar $\tau$ at the LHC and a future LC (at Run II of the Tevatron a discovery reach of only up to $\sim 150 \mathrm{GeV}$ is expected [95]). In the mSUGRA and the mAMSB scenarios, on the other hand, much larger masses of the scalar $\tau$ leptons are possible, and their discovery in the scenario considered here is not guaranteed at the next generation of colliders.

\section{Conclusions}

We have analyzed the three most prominent soft SUSY-breaking scenarios, mSUGRA, mGMSB and mAMSB, regarding their phenomenology in the Higgs sector. We have discussed the constraints arising from the exclusion limits in the Higgs sector recently obtained at LEP and the possible implications in the situation where the excess of events observed at LEP is interpreted as a signal of the light or heavy neutral $\mathcal{C P}$-even MSSM Higgs boson with mass of about $115 \mathrm{GeV}$.

In order to obtain the predictions for the Higgs sector in the three scenarios, we have combined the two-loop RG calculations, employed to derive the low-energy mass 
spectrum from the high-energy parameters in mSUGRA, mGMSB and mAMSB, with Feynman-diagrammatic results up to two-loop order for the Higgs boson spectrum and the effective mixing angle in the Higgs sector. The possible values for the low-energy mass spectrum have been obtained by scanning over the fundamental (high-energy) parameters in the scenarios. In addition to the constraints on the MSSM Higgs sector from the Higgs search at LEP, which is the main issue in this paper, we have taken into account some further phenomenological constraints on the low-energy mass spectrum. The lower bounds on SUSY particle masses obtained from the searches at LEP2 and the Tevatron have been incorporated as well as the constraints from electroweak precision observables. We have assumed $\mathcal{C P}$-invariance and conservation of $R$-parity and have discarded models giving rise to charge and color breaking minima in the scalar potential or violating the condition for radiative electroweak symmetry breaking. We have furthermore imposed a mild "naturalness" upper bound on the masses of the scalar quarks and the gluino of $1.5-2 \mathrm{TeV}$.

As upper bound on the mass of the lightest $\mathcal{C P}$-even Higgs boson (for $m_{t}=175 \mathrm{GeV}$ ) we have found $m_{h} \lesssim 124,119$ and $122 \mathrm{GeV}$ in the mSUGRA, mGMSB and mAMSB scenario, respectively. In these scenarios the $\tan \beta$ values are excluded up to $\tan \beta \gtrsim 3.3,4.6$ and 3.2 , respectively. The upper bound on $m_{h}$ in the three scenarios is significantly reduced compared to the unconstrained MSSM. This decrease in the upper bound on $m_{h}$ is in particular related to the restrictions imposed on the mixing in the scalar top sector by the underlying structure of the three scenarios. We have furthermore investigated the Higgs couplings to vector bosons and fermions (in particular the $b$ quark). Using these results, we have discussed in which parameter regions a non SM-like behavior of the Higgs production and decay processes is possible in the three scenarios.

The set of models that passed all constraints (called "case (I)" in our terminology), was then further analyzed in view of whether they permit the interpretation of the excess of events observed at LEP2 as a signal of the light $\mathcal{C} \mathcal{P}$-even Higgs boson ("case (II)") or the heavy $\mathcal{C P}$-even Higgs boson ("case (III)") with a mass of $115 \pm 2 \mathrm{GeV}$ and SM-like couplings.

While the interpretation of the LEP excess as production of the light $\mathcal{C} \mathcal{P}$-even Higgs boson is possible in all three scenarios, the interpretation as a signal of the heavy $\mathcal{C} \mathcal{P}$ even Higgs boson is only possible in the mSUGRA scenario in a small parameter region with $50 \lesssim \tan \beta \lesssim 55$, which is constrained from the Higgs search results of Run I of the Tevatron and is also close to the region where no radiative electroweak symmetry breaking occurs.

Assuming the interpretation of the LEP excess as a Higgs signal in the MSSM (according to cases (II) and (III)), we have analyzed the restrictions on the parameter space of the fundamental parameters in the three scenarios. We have furthermore investigated the corresponding spectra of the SUSY particles in these scenarios in view of the SUSY searches at Run II of the Tevatron, the LHC and an $e^{+} e^{-} \mathrm{LC}$ with center of mass energy of up to $1 \mathrm{TeV}$. While for the scenario studied here the Tevatron has only a limited chance to observe SUSY particles, the LHC can always cover the scalar tops and bottoms (the latter is related to the naturalness condition imposed on the models in our analysis). A LC offers very good prospects for gaugino and slepton production. We find that at least a part of the gaugino and slepton spectrum should be accessible at at LC with center of 
mass energy of up to $1 \mathrm{TeV}$ in all three scenarios.

\section{Acknowledgements}

We thank K. Olive for helpful communications concerning the comparison of our results. We furthermore thank L. Covi for helpful discussion on astro-physical constraints. S.S. has been supported by the DOE grant DE-FG03-92-ER-40701. A.D. would like to acknowledge financial support from the Network RTN European Program HPRN-CT-2000-0014 "Physics Across the Present Energy Frontier: Probing the Origin of Mass".

\section{References}

[1] G. Kane, C. Kolda and J. Wells, Phys. Rev. Lett. 70 (1993) 2686, hep-ph/9210242;

J. Espinosa and M. Quirós, Phys. Lett. B 302 (1993) 51, hep-ph/9212305.

[2] ALEPH collaboration, R. Barate et al., Phys. Lett. B 495 (2000) 1, hep-ex/0011045; L3 collaboration, M. Acciarri et al., Phys. Lett. B 495 (2000) 18, hep-ex/0011043; DELPHI collaboration, P. Abreu et al., Phys. Lett. B 499 (2001) 23, hep-ex/0102036; OPAL collaboration, G. Abbiendi et al., Phys. Lett. B 499 (2001) 38, hepex/0102036.

For a preliminary compilation of the LEP data presented on Nov. 3rd, 2000, see:

P. Igo-Kemenes, for the LEP Higgs working group,

lephiggs . web . cern. ch/LEPHIGGS/talks/index.html.

[3] G. Altarelli and G. Isidori, Phys. Lett. B 337 (1994) 141;

J.A. Casas, J.R. Espinosa and M. Quirós, Phys. Lett. B 342 (1995) 171, hepph/9409458;

T. Hambye and K. Riesselmann, hep-ph/9708416;

G. Isidori, G. Ridolfi and A. Strumia, hep-ph/0104016.

[4] S. Heinemeyer, W. Hollik and G. Weiglein, Eur. Phys. Jour. C 9 (1999) 343, hepph/9812472.

[5] S. Heinemeyer, W. Hollik and G. Weiglein, Phys. Rev. D 58 (1998) 091701, hepph/9803277; Phys. Lett. B 440 (1998) 296, hep-ph/9807423.

[6] M. Carena, J. Espinosa, M. Quirós and C. Wagner, Phys. Lett. B 355 (1995) 209, hep-ph/9504316.

[7] M. Carena, M. Quirós and C. Wagner, Nucl. Phys. B 461 (1996) 407, hepph/9508343.

[8] H. Haber, R. Hempfling and A. Hoang, Z. Phys. C 75 (1997) 539, hep-ph/9609331.

[9] R. Hempfling and A. Hoang, Phys. Lett. B 331 (1994) 99, hep-ph/9401219.

[10] R.-J. Zhang, Phys. Lett. B 447 (1999) 89, hep-ph/9808299. 
[11] J. Espinosa and R. Zhang, Nucl. Phys. B 586 (2000) 3, hep-ph/0003246.

[12] J. Espinosa and I. Navarro, hep-ph/0104047.

[13] G. Degrassi, P. Slavich and F. Zwirner, hep-ph/0105096.

[14] J. Ellis and D. Ross, Phys. Lett. B 506 (2001) 331, hep-ph/0012067.

[15] M. Carena, S. Heinemeyer, C. Wagner and G. Weiglein, hep-ph/9912223.

[16] H.P. Nilles, Phys. Lett. B 115 (1982) 193; Nucl. Phys. B 217 (1983) 366.

A.H. Chamseddine, R. Arnowitt and P. Nath, Phys. Rev. Lett. 49 (1982) 970;

R. Barbieri, S. Ferrara and C.A. Savoy, Phys. Lett. B 119 (1982) 343;

H.P. Nilles, M. Srednicki and D. Wyler, Phys. Lett. B 120 (1983) 346.

E. Cremmer, P. Fayet and L. Girardello, Phys. Lett. B 122 (1983) 41.

S. Ferrara, L. Girardello and H.P. Nilles, Phys. Lett. B 125 (1983) 457. L. Hall, J. Lykken and S. Weinberg, Phys. Rev. D 27 (1983) 2359;

S.K. Soni and H.A. Weldon, Phys. Lett. B 126 (1983) 215.

R. Arnowitt, A.H. Chamseddine and P. Nath, Nucl. Phys. B 227 (1983) 121.

For more details see, S. Weinberg, "The quantum theory of fields. Vol. 3: Supersymmetry," Cambridge University Press (2000).

[17] For reviews see also,

H.-P. Nilles, Phys. Rept. 110 (1984) 1;

H.E. Haber and G.L. Kane, Phys. Rept. 117 (1985) 75;

A.B. Lahanas and D.V. Nanopoulos, Phys. Rept. 145 (1987) 1;

S.P. Martin, in "Perspectives on supersymmetry", ed. G. Kane, hep-ph/9709356 (zippy.physics.niu.edu/primer.shtml).

[18] For a review, see G.F. Giudice and R. Rattazzi, Phys. Rept. 322 (1999) 419, hepph/9801271.

[19] L. Randall and R. Sundrum, Nucl. Phys. B 557 (1999) 79, hep-th/9810155.

[20] G.F. Giudice, M.A. Luty, H. Murayama and R. Rattazzi, JHEP 9812 (1998) 027, hep-ph/9810442.

[21] T. Gherghetta, G.F. Giudice, J.D. Wells, Nucl. Phys. B 559 (1999) 27, hep$\mathrm{ph} / 9904378$.

[22] J.A. Bagger, K. Matchev, D.M. Pierce, R. Zhang, Phys. Rev. D 55 (1997) 3188, hep-ph/9609444.

[23] K.T. Matchev and D.M. Pierce, Phys. Lett. B 445 (1999) 331, hep-ph/9805275;

W. de Boer, hep-ph/9808448.

[24] T.A. Kaeding and S. Nandi, hep-ph/9906342.

[25] S. Su, Nucl. Phys. B 573 (2000) 87, hep-ph/9910481. 
[26] A. Dedes, S. Heinemeyer, P. Teixeira-Dias and G. Weiglein, Jour. Phys. G 26 (2000) 582, hep-ph/9912249.

[27] S. Ambrosanio, S. Heinemeyer and G. Weiglein, in hep-ph/0002191 and hep$\mathrm{ph} / 0005142$.

[28] J. Ellis, G. Ganis, D.V. Nanopoulos and K.A. Olive, Phys. Lett. B 502 (2001) 171, hep-ph/0009355.

[29] M. Carena, H. Haber, S. Heinemeyer, W. Hollik, C. Wagner and G. Weiglein, Nucl. Phys. B 580 (2000) 29, hep-ph/0001002.

[30] S. Heinemeyer, W. Hollik and G. Weiglein, hep-ph/9910283.

[31] J. Espinosa and R. Zhang, JHEP 0003 (2000) 026, hep-ph/9912236.

[32] S. Heinemeyer, W. Hollik and G. Weiglein, Comp. Phys. Comm. 1242000 76, hepph/9812320; hep-ph/0002213.

The codes are accessible via www.feynhiggs.de .

[33] A. Dabelstein, Nucl. Phys. B 456 (1995) 25, hep-ph/9503443; Z. Phys. C 67 (1995) 495, hep-ph/9409375.

[34] The LEP working group for Higgs boson searches, LHWG Note 2001-2, lephiggs . web . cern . ch/LEPHIGGS.

[35] L. Girardello and M.T. Grisaru, Nucl. Phys. B 194 (1982) 65.

[36] For a review, see H. Dreiner, hep-ph/9707435.

[37] G. Bhattacharyya, Nucl. Phys. Proc. Suppl. 52 A (1997) 83, hep-ph/9608415.

[38] L.E. Ibañez and G.G. Ross, Phys. Lett. 110 (1982) 215;

K. Inoue, A. Kakuto, H. Komatsu and S. Takeshita, Progr. Theor. Phys. 68 (1982) 927; Progr. Theor. Phys. 71 (1984) 413;

J. Ellis, D.V. Nanopoulos and K. Tamvakis, Phys. Lett. B 121 (1983) 123;

L.E. Ibañez, Nucl. Phys. B 218 (1983) 514;

L. Alvarez-Gaumé, J. Polchinski and M. Wise, Nucl. Phys. B 221 (1983) 495;

J. Ellis, J.S. Hagelin, D.V. Nanopoulos and K. Tamvakis, Phys. Lett. B 125 (1983) 275 ;

L. Alvarez-Gaumé, M. Claudson and M. Wise, Nucl. Phys. B 207 (1982) 96.

[39] M. Drees and M.M. Nojiri, Phys. Rev. D 45 (1992) 2482.

[40] V. Barger, M.S. Berger and P. Ohmann, Phys. Rev. D 49, (1994) 4908, hep$\mathrm{ph} / 9311269$.

[41] D.M. Pierce, J.A. Bagger, K. Matchev and R. Zhang, Nucl. Phys. B 491 (1997) 3, hep-ph/9606211. 
[42] The scalar particle spectrum and phenomenology of this model has been studied in A. Dedes and A.E. Faraggi, Phys. Rev. D 62 (2000) 016010, hep-ph/9907331.

[43] D. Matalliotakis and H.-P. Nilles, Nucl. Phys. B 435 (1995) 115, hep-ph/9407251;

A. Lleyda and C. Munoz, Phys. Lett. B 317 (1993) 82, hep-ph/9308208;

N. Polonsky and A. Pomarol, Phys. Rev. D 51 (1995) 6532, hep-ph/9410231.

[44] Part. Data Group, Eur. Phys. Jour. C 15 (2000) 1.

[45] S. Martin and M. Vaughn, Phys. Lett. B 318 (1993) 331, hep-ph/9308222.

[46] H. Arason, D.J. Castano, B.Keszthelyi, S.Mikaelian, E.J. Piard, P. Ramond and B.D. Wright, Phys. Rev. D 46 (1992) 3945.

[47] A. Dedes, A.B. Lahanas and K. Tamvakis, Phys. Rev. D 53, 3793 (1996), hepph/9504239.

[48] M. Dine, W. Fischler, M. Srednicki, Nucl. Phys. B 189 (1981) 575;

S. Dimopoulos, S. Raby, Nucl. Phys. B 192 (1981) 353;

M. Dine, W. Fischler, Phys. Lett. B 110 (1982) 227;

M. Dine, M. Srednicki, Nucl. Phys. B 202 (1982) 238;

M. Dine, W. Fischler, Nucl. Phys. B 204 (1982) 346;

L. Alvarez-Gaumé, M. Claudson, M.B. Wise, Nucl. Phys. B 207 (1982) 96;

C.R. Nappi, B.A. Ovrut, Phys. Lett. B 113 (1982) 175;

S. Dimopoulos, S. Raby, Nucl. Phys. B 219 (1983) 479.

[49] M. Dine, A.E. Nelson, Phys. Rev. D 48 (1993) 1277, hep-ph/9303230;

M. Dine, A.E. Nelson, Y. Shirman, Phys. Rev. D 51 (1995) 1362, hep-ph/9408384;

M. Dine, A.E. Nelson, Y. Nir, Y. Shirman, Phys. Rev. D 53 (1996) 2658, hepph/9507378.

[50] S. Dimopoulos, S. Thomas, J.D. Wells, Phys. Rev. D 54 (1996) 3283, hepph/9604452;

Nucl. Phys. B 488 (1997) 39, hep-ph/9609434.

[51] S. Ambrosanio, G.D. Kribs, S.P. Martin, Phys. Rev. D 56 (1997) 1761, hepph/9703211.

[52] S. Ambrosanio, G.A. Blair, Eur. Phys. Jour. C 12 (2000) 287, hep-ph/9905403.

[53] S. Ambrosanio et al., JHEP 0101 (2001) 014, hep-ph/0010081.

[54] P. Fayet, Phys. Lett. B 70 (1977) 461; Phys. Lett. B 86 (1979) 272; Phys. Lett. B 175 (1986) 471 and in "Unification of the fundamental particle interactions", eds. S. Ferrara, J. Ellis, P. van Nieuwenhuizen (Plenum, New York, 1980) p. 587.

[55] An updated, generalized and Fortran-linked version of the program used in Ref. [51]. It generates minimal and non-minimal GMSB and SUGRA models. For inquiries about this software package, please send e-mail to Sandro.Ambrosanio@bancaroma.it. 
[56] K. Jacobs, talk given at the XXXVI Rencontres de Moriond, 11th of March 2001, Moriond, France.

[57] A. Pomarol and R. Rattazzi, JHEP 9905 (1999) 013, hep-ph/9903448.

[58] Z. Chacko, M.A. Luty and E. Ponton, JHEP 0004 (2000) 001, hep-ph/9905390.

[59] B.C. Allanach and A. Dedes, JHEP 0006 (2000) 017, hep-ph/0003222.

[60] E. Katz, Y. Shadmi and Y. Shirman, JHEP 9908 (1999) 015, hep-ph/9906296 .

[61] S. Heinemeyer, W. Hollik and G. Weiglein, Eur. Phys. Jour. C 16 (2000) 139, hep$\mathrm{ph} / 0003022$.

[62] S. Heinemeyer, W. Hollik, J. Rosiek and G. Weiglein, Eur. Phys. Jour. C 19 (2001) 535, hep-ph/0102081.

[63] A. Dedes, unpublished.

[64] A. Dedes, S. Heinemeyer and G. Weiglein, "FeynSSG: A program for the minimal supergravity and Higgs spectrum", in preparation. The code will be accessible via www . feynhiggs.de .

[65] P. Chankowski, S. Pokorski and J. Rosiek, Phys. Lett. B 286 (1992) 307; Nucl. Phys. B 423 (1994) 423, hep-ph/9303309.

[66] M. Frank, S. Heinemeyer, W. Hollik and G. Weiglein, in preparation.

[67] M. Veltman, Nucl. Phys. B 123 (1977) 89.

[68] A. Djouadi, P. Gambino, S. Heinemeyer, W. Hollik, C. Jünger and G. Weiglein, Phys. Rev. Lett. 78 (1997) 3626, hep-ph/9612363; Phys. Rev. D 57 (1998) 4179, hep-ph/9710438;

S. Heinemeyer and G. Weiglein, hep-ph/0102317.

[69] LEP Fest, Oct.9-11, 2000, Talks given by F. Cerutti (ALEPH), M. Kienzle (L3), R. Hemingway (OPAL), V. Hedberg (DELPHI).

[70] C. Pagliarone, SUSY Searches at the Tevatron Collider, Proceedings of 13th Topical Conference on Hadron Collider Physics, Mumbai, India, 14-20 Jan 1999, hepex/0011016.

[71] I. Iashvili, hep-ex/0007001.

[72] J.B. de Vivie, hep-ex/9911032.

[73] S. Heinemeyer, W. Hollik and G. Weiglein, JHEP 0006 (2000) 009, hep-ph/9909540.

[74] A. Riotto and E. Roulet, Phys. Lett. B 377 (1996) 60, hep-ph/9512401.

[75] A. Kusenko, P. Langacker and G. Segre, Phys. Rev. D 54 (1996) 5824, hepph/9602414. 
[76] S. Abel, C.A. Savoy, Phys. Lett B 444 (1998) 119, hep-ph/9809498;

S. Abel, T. Falk, Phys. Lett. B 444 (1998) 427, hep-ph/9810297;

S. Abel and B.C. Allanach, JHEP 0007 (2000) 037, hep-ph/9909448.

[77] For an overview on bounds see for example

S. Sarkar, Rept. Prog. Phys. 59 (1996) 1493, hep-ph/9602260.

[78] CLEO Collaboration, M.S. Alam et al., Phys. Rev. Lett. 74 (1995) 2885 as updated in S. Ahmed et al., CLEO CONF 99-10;

K. Abe et al., Belle Collaboration, hep-ex/0103042.

[79] H.N. Brown et al., Muon $g_{\mu}-2$ Collaboration, Phys. Rev. Lett. 86 (2001) 2227, hep-ex/0102017;

A. Czarnecki and W. J. Marciano, Phys. Rev. D 64 (2001) 013014, hep-ph/0102122.

[80] G. Kane, S. King and L.-T. Wang, hep-ph/0010312;

A. Bottino, N. Fornengo and S. Scopel, hep-ph/0012377;

J. Ellis, D.V. Nanopoulos and K.A. Olive, hep-ph/0102331.

[81] J. Ellis, S. Heinemeyer, K.A. Olive and G. Weiglein, to appear in Phys. Lett. B, hep-ph/0105061.

[82] J.L. Feng and T. Moroi, Phys. Rev. D 61 (2000) 095004, hep-ph/9907319;

J.L. Feng, K.T. Matchev and T. Moroi, Phys. Rev. Lett. 84 (2000) 2322, hepph/9908309; Phys. Rev. D 61 (2000) 075005, hep-ph/9909334.

[83] F. Gabbiani, E. Gabrielli, A. Masiero and L. Silvestrini, Nucl. Phys. B 477 (1996) 321, hep-ph/9604387;

F. Borzumati, C. Greub, T. Hurth and D. Wyler, Phys. Rev. D 62 (2000) 075005, hep-ph/9911245.

[84] J. Ellis, J. Hagelin, D. Nanopoulos, K. Olive and M. Srednicki, Nucl. Phys. B238 (1984) 453;

H. Goldberg, Phys. Rev. Lett. 50 (1983) 1419;

J. Ellis, T. Falk, G. Ganis, K. Olive and M. Srednicki, Phys. Lett. B 510 (2001) 236, hep-ph/0102098.

[85] D. Lyth and E. Stewart, Phys. Rev. D 53 (1996) 1784, hep-ph/9510204.

[86] L. Covi, J. Kim and L. Roszkowski, Phys. Rev. Lett. 82 (1999) 4180, hep-ph/9905212.

[87] R. Barate et al. [ALEPH Collaboration], Phys. Lett. B 499 (2001) 53, hepex/0010062.

[88] T. Affolder et al., [CDF Collaboration] Phys. Rev. Lett. D 86 (2001) 4472, hepex/0010052.

[89] L.J. Hall, R. Rattazzi and U. Sarid, Phys. Rev. D 50 (1994) 7048, hep-ph/9306309. 
[90] M. Carena, S. Mrenna and C. Wagner, Phys. Rev. D 60 (1999) 075010, hepph/9808312; Phys. Rev. D 62 (2000) 055008, hep-ph/9907422;

H. Eberl, K. Hidaka, S. Kraml, W. Majerotto and Y. Yamada, Phys. Rev. D 62 (2000) 055006, hep-ph/9912463;

M. Carena, D. Garcia, U. Nierste and C. Wagner, Nucl. Phys. B 577 (2000) 88, hep-ph/9912516.

[91] S. Heinemeyer, W. Hollik and G. Weiglein, Phys. Lett. B 455 (1999) 179, hepph/9903404.

[92] J. Ellis, G. Ganis, D.V. Nanopoulos and K.A. Olive, hep-ph/0009355;

J. Ellis, hep-ex/0011086.

[93] I. Hinchliffe and P. Richardson, hep-ph/0106212.

[94] A. Belyaev, D. Garcia, J. Guasch and J. Sola, hep-ph/0105053.

[95] S. Abel et al. [SUGRA Working Group Collaboration], Report of the SUGRA working group for Run II of the Tevatron, hep-ph/0003154 and references therein;

R. Culbertson et al., hep-ph/0008070, and references therein.

[96] ATLAS Collaboration, Detector and Physics Performance Technical Design Report, CERN/LHCC/99-15 (1999), see: atlasinfo.cern.ch/Atlas/GROUPS/PHYSICS/TDR/access.html .

[97] CMS Collaboration, see: cmsinfo.cern.ch/Welcome.html/CMSdocuments/CMSplots/ .

[98] TESLA TDR Part 3: "Physics at an $e^{+} e^{-}$Linear Collider", eds. R.D. Heuer, D. Miller, F. Richard and P.M. Zerwas, hep-ph/0106315, see: tesla.desy.de .

[99] T. Abe et al. [American Linear Collider Working Group Collaboration], Resource book for Snowmass 2001, hep-ex/0106055, hep-ex/0106056, hep-ex/0106057, hepex/0106058.

[100] J.L. Feng and T. Moroi, Phys. Rev. D 61 (2000) 095004, hep-ph/9907319.

[101] J.L. Feng, T. Moroi, L. Randall, M. Strassler and S. Su, Phys. Rev. Lett. 83 (1999) 1731, hep-ph/9904250.

[102] C.H. Chen, M. Drees and J. Gunion, Phys. Rev. Lett. 76 (1996) 2002, hepph/9512230; E: Phys. Rev. D 55 (1997) 330, hep-ph/9902309;

J. Gunion and S. Mrenna, Phys. Rev. D 62 (2000) 015002, hep-ph/9906270. 